\title{
Radionuclide Data and Calculations and Loss-On-Ignition, $X$-Ray Fluorescence, and ICP-AES Data from Cores in Catchments of the Animas River, Colorado
}

Data Series 382 



\section{Radionuclide Data and Calculations and Loss-On-Ignition, X-Ray Fluorescence, and ICP-AES Data from Cores in Catchments of the Animas River, Colorado}

By Stanley E. Church, Cyndi A. Rice, and Marci E. Marot

Data Series 382 


\section{U.S. Department of the Interior DIRK KEMPTHORNE, Secretary}

\section{U.S. Geological Survey \\ Mark D. Myers, Director}

\section{U.S. Geological Survey, Reston, Virginia: 2008}

For product and ordering information:

World Wide Web: http://www.usgs.gov/pubprod

Telephone: 1-888-ASK-USGS

For more information on the USGS--the Federal source for science about the Earth, its natural and living resources, natural hazards, and the environment:

World Wide Web: http://www.usgs.gov

Telephone: 1-888-ASK-USGS

Any use of trade, product, or firm names is for descriptive purposes only and does not imply endorsement by the U.S. Government.

Although this report is in the public domain, permission must be secured from the individual copyright owners to reproduce any copyrighted materials contained within this report.

Suggested citation:

Church, S.E., Rice, C.A., and Marot, M.E., 2008, Radionuclide data and calculations and loss-on-ignition, X-ray fluorescence, and ICP-AES data from cores in catchments of the Animas River, Colorado: U.S. Geological Survey Data Series 382, 20 p. 


\section{Contents}

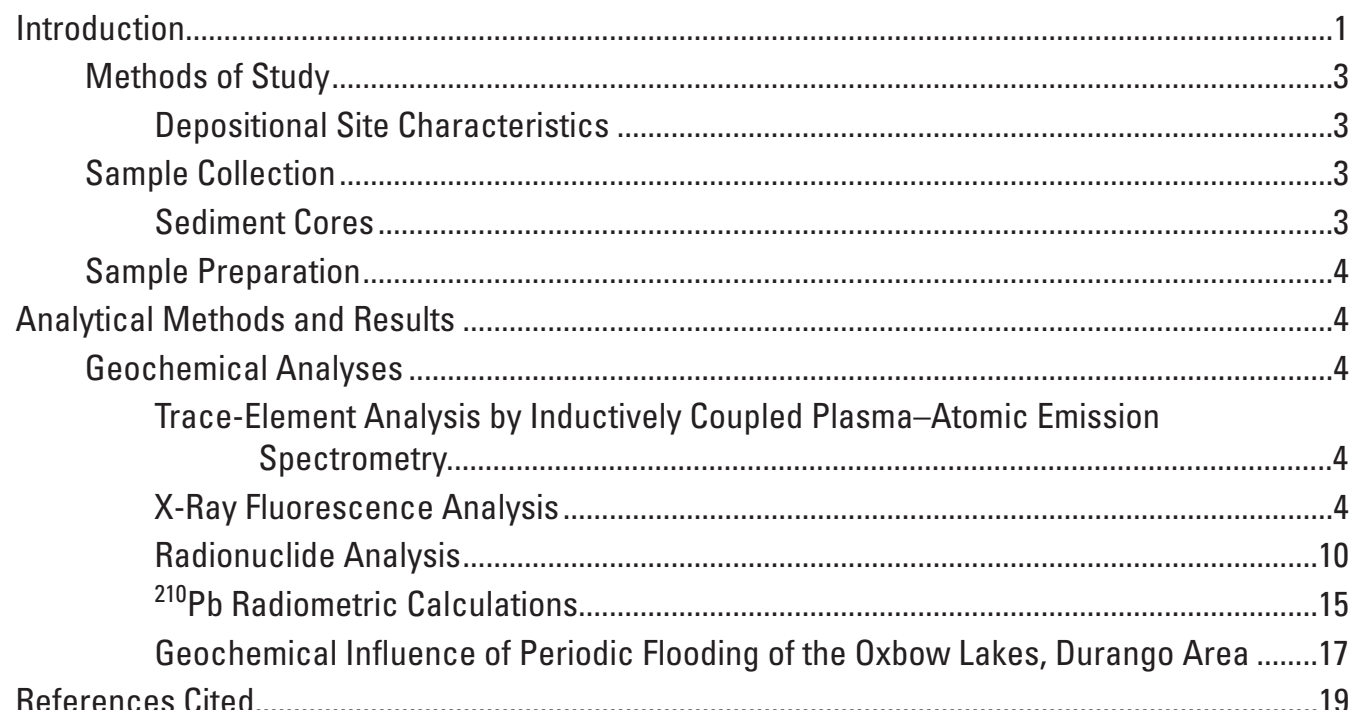

\section{Figures}

1. Map of the Animas River watershed, Colorado, showing relationship of two detailed study areas to Animas River.

2. 1973 aerial photograph showing localities sampled in oxbow lakes, Durango, Colorado

3. 1973 aerial photograph showing localities of sampled abandoned beaver ponds, Mineral Creek drainage, Colorado

4-6. ${ }^{210} \mathrm{~Pb}$ decay curve for:

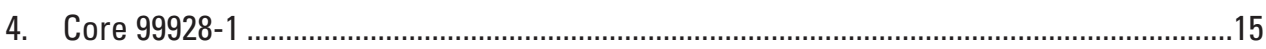

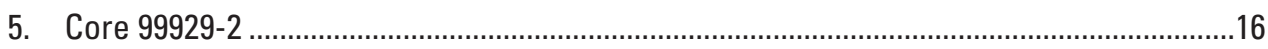

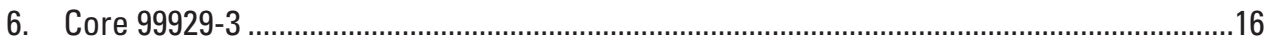

\section{Tables}

1. Sample localities and data on cores, Animas River watershed, Colorado .........................4

2-6. Core descriptions, depths, and geochemical data from:

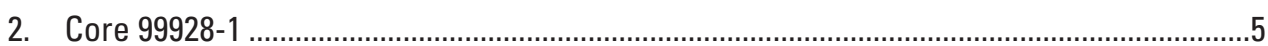

3. Core 97ABS300

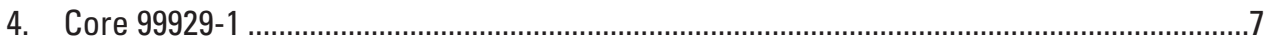

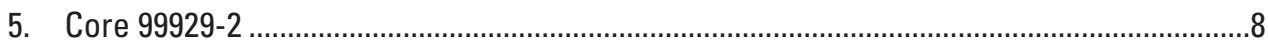

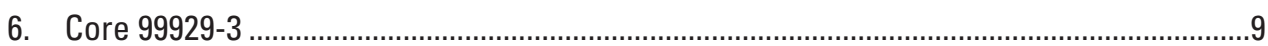

7-10. Sample depths, loss-on-ignition, radionuclide activity, flux, and inventory data and ${ }^{210} \mathrm{~Pb}$ age calculations for:

7. Core 99928-1 


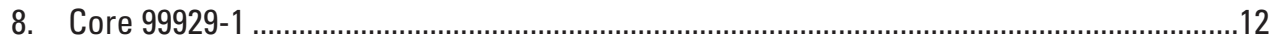

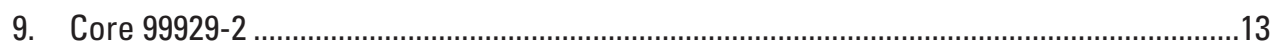

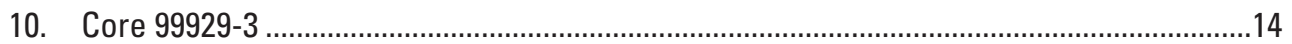

11. Discharge records from NWIS gage 09361500, 32nd St. Bridge, Durango, Colo ............18 


\title{
Radionuclide Data and Calculations and Loss-On-Ignition, $X$-Ray Fluorescence, and ICP-AES Data from Cores in Catchments of the Animas River, Colorado
}

\author{
By Stanley E. Church, Cyndi A. Rice, and Marci E. Marot
}

\section{Introduction}

The U.S. Departments of Agriculture and Interior (USDA-DOI) Abandoned Mine Lands (AML) Initiative is focused on the evaluation of the effect of past mining practices on the water quality and the riparian and aquatic habitats of impacted stream reaches downstream from historical mining districts located primarily on Federal lands. This problem is manifest in the eleven western states (west of longitude $102^{\circ}$ ) where the majority of hardrock mines that had past production are located on Federal lands (Ferderer, 1996; USDA Forest Service and Bureau of Land Management, 2007). In areas of temperate climate and moderate to heavy precipitation, the effects of rapid chemical and physical weathering of sulfides exposed on mine-waste dumps and acidic drainage from mines have resulted in elevated metal concentrations in the stream water and stream-bed sediment. The result of these mineral weathering processes has an unquantified impact on the quality of the water and the aquatic and riparian habitats that may limit their recreational resource value. One of the confounding factors in these studies is the determination of the component of metals derived from hydrothermally altered but unmined portions of these drainage basins (Runnells and others, 1992).

Several watersheds have been studied to evaluate the effects of acid mine drainage (AMD) and acid rock drainage (ARD) on the near-surface environment (U.S. Geological Survey, 1997). The Animas River watershed in southwestern Colorado (fig. 1) contains a large number of past-producing metal mines (Church and others, 2007) that have affected the watershed. Beginning in October 1996, the U.S. Geological Survey (USGS) began a collaborative study of these effects under the USGS-AML Initiative (Buxton and others, 1997). In this report, we present the radionuclide and geochemical analytical results of sediment coring during 1997-1999 from two cores from oxbow lakes $0.5 \mathrm{mi}$. upstream from the 32nd Street Bridge near Durango, Colo. (fig. 2), and from three cores from beaver ponds within the Mineral Creek drainage basin near Silverton, Colo. (fig. 3).

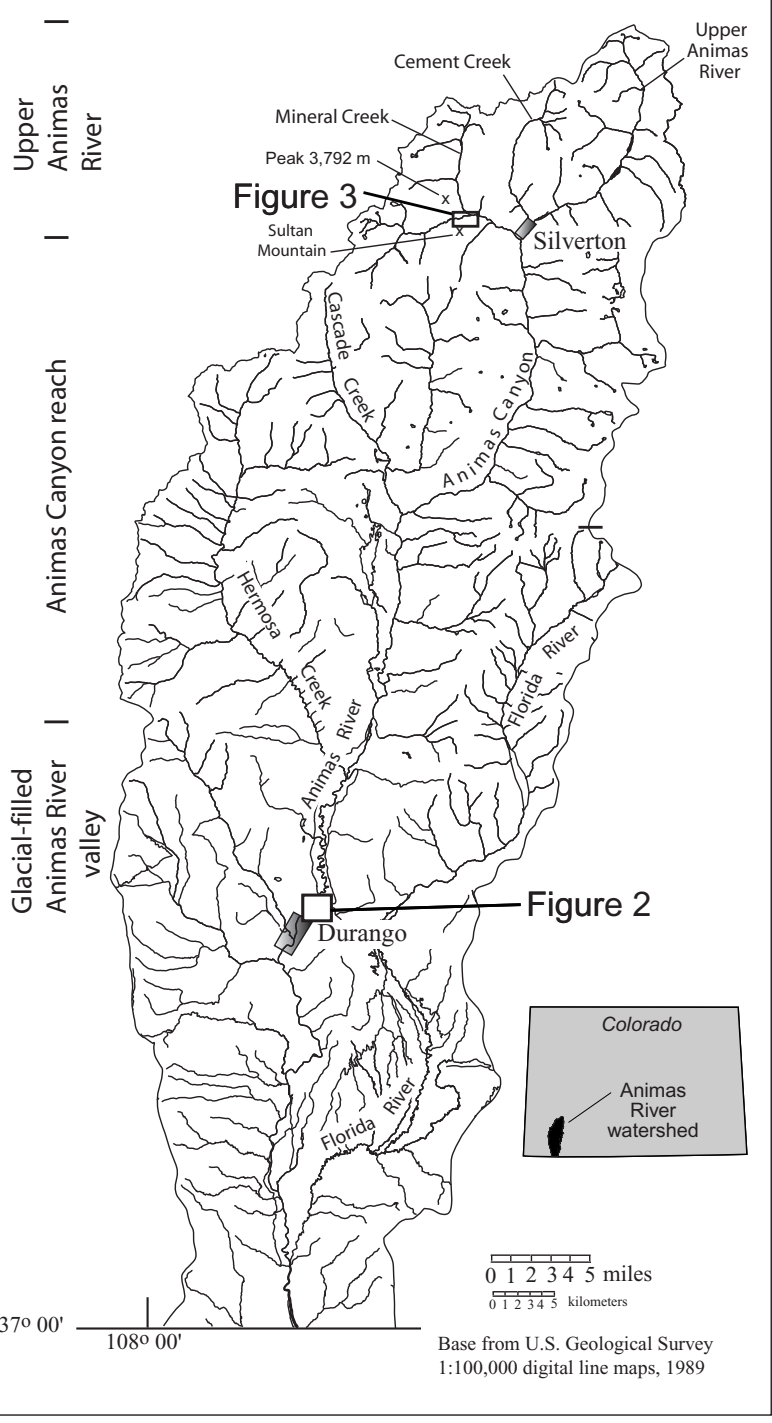

Figure 1. Map of the Animas River watershed in Colorado showing the relationship of the mineralized headwaters area of the watershed north of Silverton to areas studied near Durango (fig. 2) and Mineral Creek west of Silverton (fig. 3). 


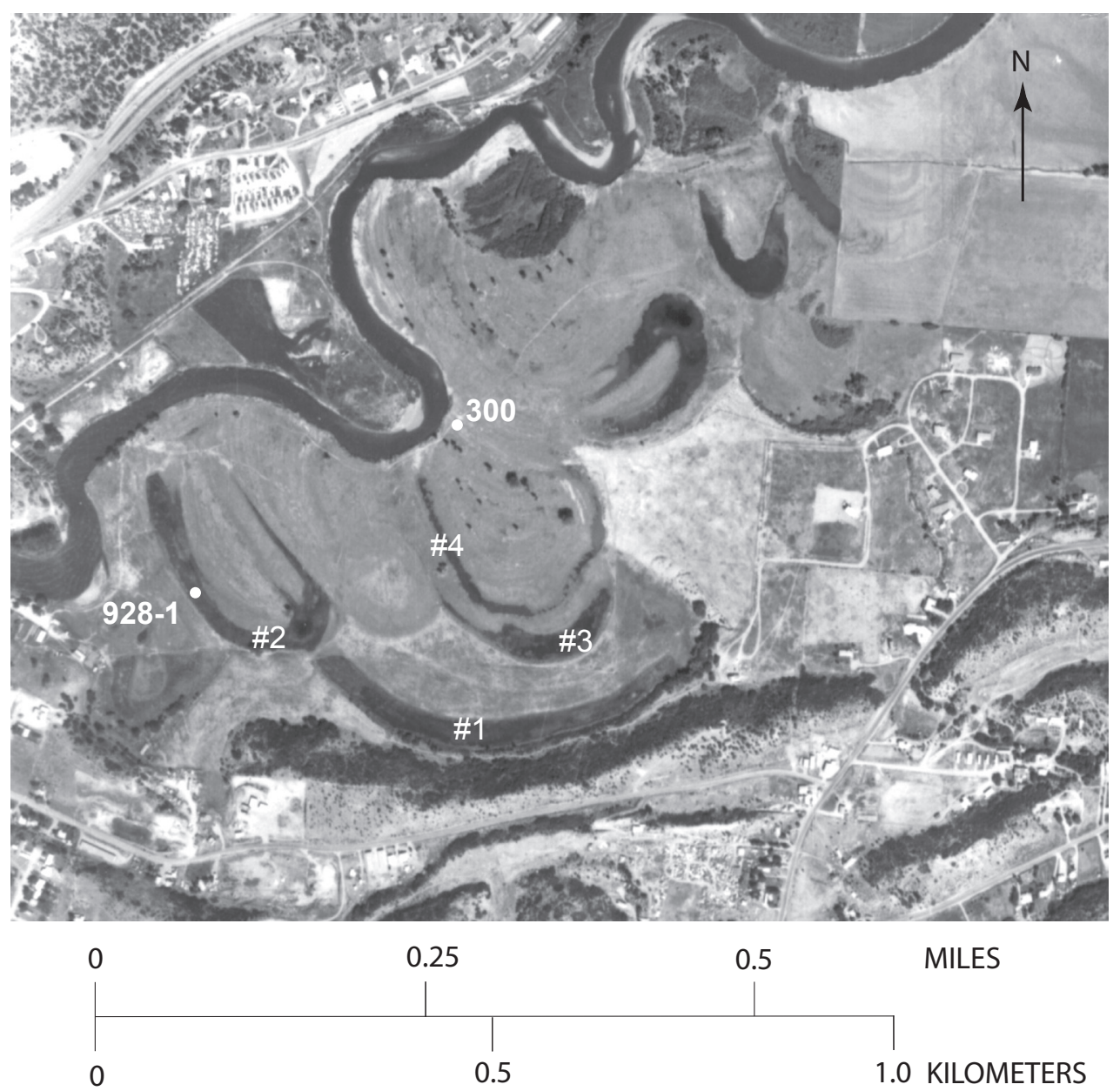

Figure 2. Enlargement of the 1973 aerial photograph showing the active meander of the Animas River and oxbow lakes sampled just north of Durango, Colo. Downstream is to the left; north is at the top of the photograph. Core localities are shown for samples taken for flux determinations (tables 1 and 2). Oxbow lakes are numbered in the order they formed: oxbow lake \#1 is the oldest, oxbow lake \#4 (core 97ABS300; 300 on figure) the youngest. Oxbow lake \#4 was active when the initial topographic mapping was completed in 1896 (U.S. Geological Survey, 1898). Core locality for dated core (99928; 928-1 on figure) shown in oxbow lake \#2. . 


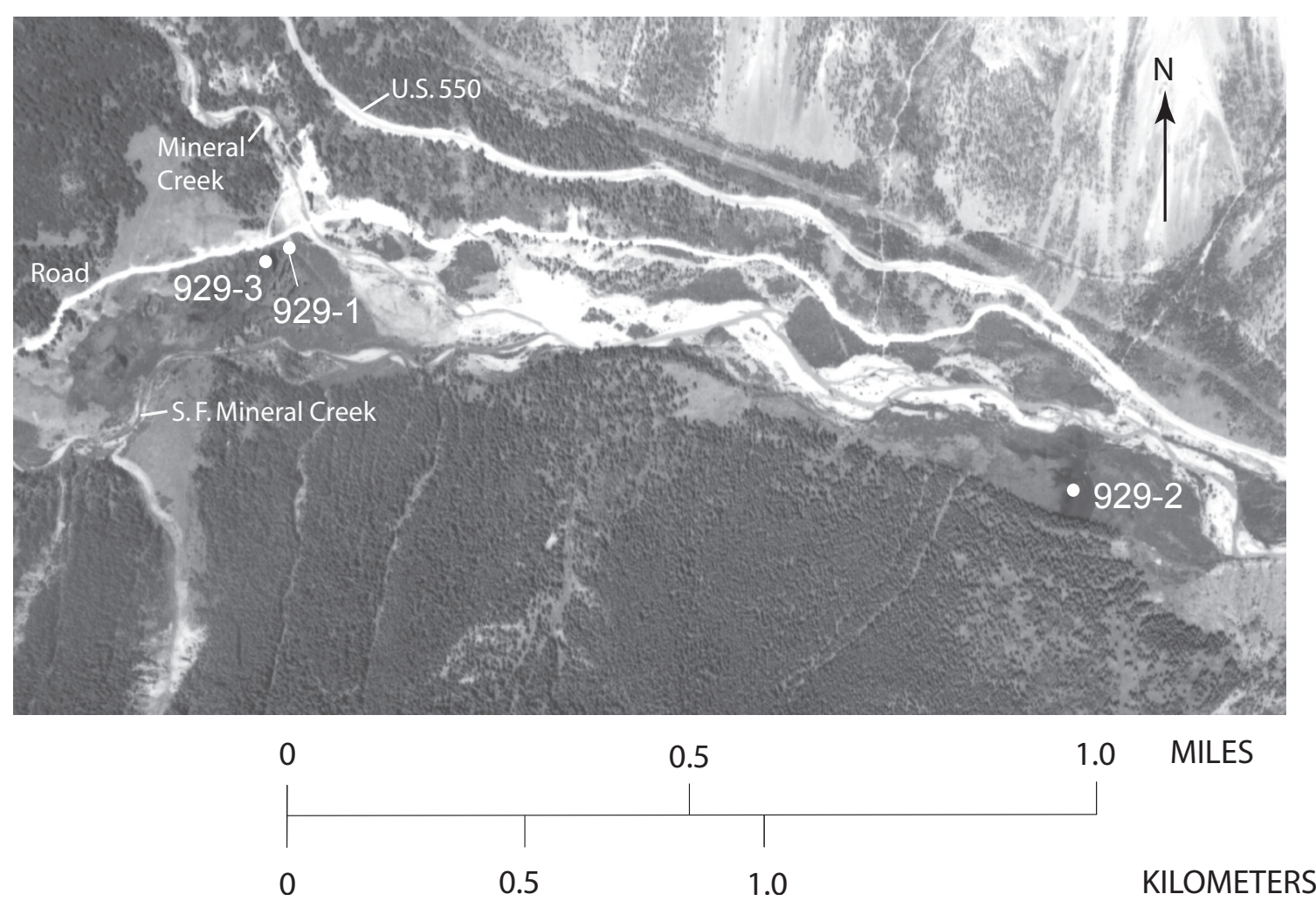

Figure 3. Enlargement of the 1973 aerial photograph taken near the confluence of Mineral Creek with the South Fork Mineral Creek showing localities of abandoned beaver ponds sampled west of Silverton, Colo. Downstream is to the right; north is at the top of the photograph. Elevations of both sets of beaver ponds are above flood stage, and the ponds are ground-water fed. Sultan Mountain, composed largely of unmineralized granodiorite, is south of site $929-2$, and peak $3,792 \mathrm{~m}$, underlain by a porphyry copper-molybdenum deposit, is northwest of site 929-3. Core numbers have been abbreviated on figure.

\section{Methods of Study}

\section{Depositional Site Characteristics}

Samples for this study were collected from two different depositional environments, both of which are geomorphologically distinct. Many stream reaches in the headwaters are downcutting, have broad to narrow riparian zones (fig. 3), and often support an active beaver population. Beaver ponds in the upper watershed in the Mineral Creek drainage basin are built on the sides of the riparian zone, trapping ground water before it enters the streams. The downstream reach of the Animas River has numerous meanders and has created many generations of oxbow lakes (fig. 2). The depositional environments of beaver ponds and oxbow lakes provide ideal sedimentary repositories that preserve a stratigraphic and chronologic record of environmental change in the watershed, and both depositional environments have low sedimentation rates and significant spring-fed ground-water components in their water budgets (Fey and others, 2000). In this study, we report the results of geochemical, lead $(\mathrm{Pb})$ isotopic, and radionuclide data from these young sediment catchments to document the recent changes $(<200 \mathrm{yr})$ in geochemistry caused by historical mining.

\section{Sample Collection}

\section{Sediment Cores}

Sediment cores were collected by driving and subsequently extracting sections of $10-\mathrm{cm}$-diameter PVC pipe that had previously been acid-cleaned and water-rinsed into the sediment (sites 99928-1; 99929-1 through 99929-3). These cores were subsequently split lengthwise and sampled in 1-2 $\mathrm{cm}$ intervals, taking care to avoid material in contact with the core liner and along the split-core surface, to give the time resolution needed for the study. Core $97 \mathrm{ABS} 300$ was a $5-\mathrm{cm}-$ diameter core that was subdivided based on stratigraphic, color, and grain-size differences (Fey and others, 2000). Core sample localities and depth intervals are in table 1. Subsamples from the cores were subsequently analyzed for radionuclides, element abundances, and loss-on-ignition. 
Table 1. Sample localities and data on cores, Animas River watershed, Colorado. [Core 97ABS300 collected by D.L. Fey and S.E. Church, property accessed by permission; other cores collected by C.W. Holmes, M.E. Marot, and S.E. Church on USDA Forest Service land. DD, decimal degrees]

\begin{tabular}{lccccc}
\hline Core no. & Date collected & Latitude DD & Longitude DD & Diameter of core (cm) & Total depth (cm) \\
99928-1 & 28-Sep-99 & 37.29992 & 107.85964 & 10 & 42 \\
$97 A B S 300$ & 16-Aug-97 & 37.30483 & 107.85519 & 5 & 238 \\
$99929-1$ & $29-S e p-99$ & 37.81767 & 107.72000 & 10 & 54 \\
$99929-2$ & $29-S e p-99$ & 37.81553 & 107.70283 & 10 & 46 \\
$99929-3$ & $29-S e p-99$ & 37.81786 & 107.72039 & 10 & 65 \\
\hline
\end{tabular}

\section{Sample Preparation}

Core subsamples were air dried. The midpoint of the depth interval for each subsample is used as the depth for that subsample. No depth corrections for compression were made on the 10-cm-diameter cores. Samples with high organic content (peat) were ashed prior to analysis (Fey and others, 2000).

Because the grain size in all samples except those from core $97 \mathrm{ABS} 300$ was predominately silt, subsamples were mixed but not ground to finer grain size. Core 97ABS300 was expanded linearly to correct for compression (Fey and others, 2000). Core compaction was determined by measuring the depth of penetration of the core casing in the field, and then measuring the length of the recovered core material. Subsamples from core 97ABS300 were air dried, sieved if the grain size exceeded that of fine sand $(0.125-0.25 \mathrm{~mm})$, and the fines retained and ground in random order to minus 100 mesh in a vertical pulverizer. The grain size and sample type are briefly described for each interval in the data tables (2-6). The peat zones in the beaver ponds are described in tables $4-6$ as matted fiber or grass.

\section{Analytical Methods and Results}

\section{Geochemical Analyses}

\section{Trace-Element Analysis by Inductively Coupled Plasma-Atomic Emission Spectrometry}

The samples were digested with a mixed-acid procedure consisting of hydrochloric, nitric, perchloric, and hydrofluoric acids ( $\mathrm{HCl}, \mathrm{HNO}_{3}, \mathrm{HClO}_{4}$, and $\mathrm{HF}$ ) (Crock and others, 1983; Briggs, 2002). This procedure is effective in dissolving most minerals, including silicates, oxides and sulfides; resistant or refractory minerals such as zircon, chromite, and some tin oxides are only partially dissolved. Previous investigations using a variety of materials support the completeness of the digestion (Church, 1981; Church and others, 1987; Wilson and others, 1994). Trace- and major-element data (tables 2-6) were determined by ICP-AES (inductively coupled plasmaatomic emission spectrometry; Briggs, 2002). ICP-AES data are reported for $\mathrm{As}, \mathrm{Cd}, \mathrm{Cu}, \mathrm{Pb}, \mathrm{Mn}, \mathrm{Ag}$, and $\mathrm{Zn}$ for all sample intervals, and for the major elements if the core subsample was not analyzed by X-ray fluorescence (Fey and others, 2000). Limits of determination for the ICP-AES total digestion multiacid method as well as a statistical summary of mean values, standard deviations, and median values for four National Institute of Standards and Technology (NIST) standard reference materials (SRM-2704, SRM-2709, SRM-2710, and SRM-2711) are given in Fey and others (1999). In addition, comparisons with certified values for these standards (NIST, 1993a,b,c,d) are in Fey and others (1999). Both analytical precision and accuracy are well within acceptable ranges of error for the method (Briggs, 2002).

\section{X-Ray Fluorescence Analysis}

Samples from cores 99928-1 (table 2), 99929-2 (table 5), and 97ABS300 (table 3) were also analyzed using wavelengthdispersive X-ray fluorescence spectrometry (Taggart and others, 1987) by a contract laboratory to obtain more reliable and accurate major-element data including $\mathrm{SiO}_{2}$. X-ray fluorescence data for $\mathrm{SiO}_{2}$ were found to be important to track the contribution of windblown mill tailings to sediment in the two small catchment basins (cores 99928-1 and 99929-2) because the ore milled at the Sunnyside Mill at Eureka during the period from 1900-1915 was predominately galena in quartz $\left(\mathrm{SiO}_{2}\right)$ veins (Jones, 2007). $\mathrm{SiO}_{2}$ is an important sedimentological and geochemical marker used in conjunction with $\mathrm{Pb}$ concentrations to determine the 1911 flood event recorded in core 97ABS300. 
Table 2. Core descriptions, depths, and geochemical data from core 99928-1.

[Core descriptions from Fey and others (2000), table 5; ICP-AES data from table 9; $x$-ray fluorescence data by contract laboratory]

$\mathrm{X}$-ray fluorescence data

ICP-AES data

\begin{tabular}{|c|c|c|c|c|c|c|c|c|c|c|c|c|c|c|c|c|c|c|c|}
\hline $\begin{array}{l}\text { Field sample } \\
\text { no. }\end{array}$ & $\begin{array}{l}\text { Sample interval } \\
\text { midpoint depth } \\
\text { (cm) }\end{array}$ & Subinterval sample description & $\begin{array}{l}\mathrm{Al}_{2} \mathrm{O}_{3} \\
\text { wt. \% }\end{array}$ & $\begin{array}{c}\mathrm{Ca0} \\
\text { wt. } \%\end{array}$ & $\begin{array}{l}\mathrm{Fe}_{2} \mathrm{O}_{3} \\
\text { wt. \% }\end{array}$ & $\begin{array}{c}\mathbf{K}_{2} \mathbf{0} \\
\text { wt. \% }\end{array}$ & $\begin{array}{l}\mathrm{MgO} \\
\text { wt. \% }\end{array}$ & $\begin{array}{l}\text { Mn0 } \\
\text { wt. \% }\end{array}$ & $\begin{array}{l}\mathrm{Na}_{2} \mathrm{O} \\
\text { wt. \% }\end{array}$ & $\begin{array}{l}\mathrm{P}_{2} \mathbf{O}_{5} \\
\text { wt. \% }\end{array}$ & $\begin{array}{c}\mathrm{SiO}_{2} \\
\text { wt.\% }\end{array}$ & $\begin{array}{r}\mathrm{TiO}_{2} \\
\text { wt. \% }\end{array}$ & $\begin{array}{c}\text { Arsenic } \\
\text { ppm }\end{array}$ & $\begin{array}{l}\text { Cadmium } \\
\text { ppm }\end{array}$ & $\begin{array}{c}\text { Copper } \\
\text { ppm }\end{array}$ & $\begin{array}{l}\text { Lead } \\
\text { ppm }\end{array}$ & $\begin{array}{c}\text { Manganese } \\
\text { ppm }\end{array}$ & $\begin{array}{c}\text { Silver } \\
\text { ppm }\end{array}$ & $\begin{array}{l}\text { Zinc } \\
\text { ppm }\end{array}$ \\
\hline $999281-1$ & 1 & light-brown silt, some grass fibers & 17.42 & 2.23 & 7.73 & 3.81 & 2.58 & 0.56 & 1.24 & 0.38 & 63.20 & 0.83 & 31 & 10 & 360 & 1,400 & 2,800 & 8 & 2,200 \\
\hline 999281-2 & 2 & light-brown silt, twigs, root mat & 17.46 & 1.69 & 7.21 & 3.70 & 2.54 & 0.44 & 1.29 & 0.34 & 64.51 & 0.83 & 32 & 8 & 360 & 1,500 & 2,400 & 9 & 2,100 \\
\hline 999281-3 & 3 & light-brown silt, twigs, root mat & 17.53 & 1.52 & 7.36 & 3.71 & 2.50 & 0.58 & 1.25 & 0.33 & 64.41 & 0.82 & 27 & 9 & 510 & 2,000 & 3,400 & 11 & 2,400 \\
\hline 999281-4 & 4 & light-brown silt, twiglets & 17.62 & 1.49 & 7.28 & 3.73 & 2.47 & 0.55 & 1.27 & 0.32 & 64.46 & 0.81 & 30 & 9 & 460 & 1,800 & 3,100 & 10 & 2,100 \\
\hline 999281-5 & 5 & light-brown silt, rootlets & 17.48 & 1.51 & 7.58 & 3.71 & 2.52 & 0.739 & 1.32 & 0.30 & 64.34 & 0.84 & 36 & 9 & 360 & 1,500 & 2,300 & 8 & 2,000 \\
\hline 999281-6 & 6 & light-brown silt, rootlets & 17.42 & 1.58 & 7.63 & 3.71 & 2.53 & 0.36 & 1.35 & 0.30 & 64.29 & 0.82 & 30 & 8 & 320 & 1,300 & 2,100 & 7 & 1,800 \\
\hline 999281-7 & 7 & light-brown silt, rootlets & 17.58 & 1.63 & 7.59 & 3.72 & 2.57 & 0.34 & 1.37 & 0.31 & 64.07 & 0.82 & 33 & 8 & 310 & 1,300 & 2,100 & 7 & 1,800 \\
\hline 999281-8 & 8 & light-brown silt, rootlets & 17.30 & 1.55 & 7.57 & 3.69 & 2.52 & 0.32 & 1.34 & 0.29 & 64.58 & 0.83 & 26 & 8 & 310 & 1,200 & 1,900 & 6 & 1,700 \\
\hline 999281-9 & 9 & light-brown silt, sparse fibers & 16.91 & 1.51 & 7.78 & 3.63 & 2.44 & 0.32 & 1.38 & 0.28 & 64.95 & 0.81 & 28 & 7 & 300 & 1,200 & 2,000 & 7 & 1,800 \\
\hline 999281-10 & 10 & light-brown silt, sparse fibers & 16.65 & 1.50 & 7.76 & 3.55 & 2.40 & 0.37 & 1.40 & 0.27 & 65.30 & 0.79 & 34 & 8 & 360 & 1,500 & 2,500 & 8 & 2,100 \\
\hline 999281-12 & 11 & light-brown silt, sparse fibers & 16.61 & 1.50 & 7.61 & 3.54 & 2.42 & 0.38 & 1.42 & 0.27 & 65.42 & 0.82 & 33 & 8 & 340 & 1,300 & 2,400 & 7 & 1,800 \\
\hline 999281-14 & 13 & light-brown silt, sparse fibers & 15.86 & 1.43 & 7.39 & 3.42 & 2.33 & 0.32 & 1.54 & 0.24 & 66.69 & 0.79 & 27 & 5 & 150 & 430 & 2,100 & 2 & 990 \\
\hline 999281-16 & 15 & light-brown silt, sparse fibers & 14.37 & 1.37 & 6.82 & 3.18 & 2.07 & 0.24 & 1.70 & 0.23 & 69.26 & 0.76 & 20 & 4 & 64 & 140 & 1,700 & $<2$ & 520 \\
\hline 999281-18 & 17 & light-brown silt, sparse fibers & 14.36 & 1.77 & 6.88 & 3.16 & 2.20 & 0.31 & 1.64 & 0.23 & 68.68 & 0.78 & 21 & 3 & 63 & 270 & 2,100 & $<2$ & 480 \\
\hline 999281-20 & 19 & light-brown silt, sparse fibers & 14.44 & 3.63 & 6.98 & 3.09 & 2.28 & 0.35 & 1.56 & 0.23 & 66.65 & 0.79 & 13 & 3 & 59 & 94 & 2,300 & $<2$ & 380 \\
\hline 999281-22 & 21 & light-brown silt and clay, blocky & 14.18 & 4.20 & 6.95 & 3.05 & 2.25 & 0.32 & 1.59 & 0.22 & 66.47 & 0.78 & 16 & 3 & 47 & 63 & 2,100 & $<2$ & 270 \\
\hline 999281-24 & 23 & $\begin{array}{l}\text { light-brown silt and clay, blocky, } \\
\text { sparse fibers }\end{array}$ & 13.80 & 4.49 & 6.59 & 2.98 & 2.22 & 0.28 & 1.59 & 0.21 & 67.07 & 0.76 & 21 & 2 & 43 & 58 & 1,900 & $<2$ & 250 \\
\hline $999281-26$ & 25 & $\begin{array}{l}\text { light-brown silt and clay, blocky, } \\
\text { sparse fibers }\end{array}$ & 13.20 & 3.26 & 5.94 & 2.97 & 2.07 & 0.24 & 1.74 & 0.19 & 69.69 & 0.71 & 18 & 2 & 44 & 53 & 1,700 & $<2$ & 200 \\
\hline $999281-28$ & 27 & light-brown silt, no clay & 12.85 & 3.10 & 5.50 & 2.96 & 1.91 & 0.19 & 1.85 & 0.18 & 70.82 & 0.64 & 14 & 2 & 33 & 48 & 1,500 & $<2$ & 180 \\
\hline 999281-30 & 29 & silt and clay, blocky & 13.21 & 3.84 & 6.01 & 2.97 & 2.07 & 0.19 & 1.76 & 0.19 & 69.07 & 0.68 & 14 & 2 & 42 & 52 & 1,400 & $<2$ & 200 \\
\hline $999281-32$ & 31 & silt and clay, blocky & 13.58 & 4.06 & 6.33 & 3.00 & 2.20 & 0.19 & 1.65 & 0.21 & 68.06 & 0.74 & 15 & 2 & 47 & 47 & 1,200 & $<2$ & 200 \\
\hline 999281-34 & 33 & silt and clay, blocky & 13.59 & 2.89 & 6.19 & 3.04 & 2.09 & 0.18 & 1.81 & 0.19 & 69.32 & 0.70 & 17 & 2 & 39 & 50 & 1,300 & $<2$ & 200 \\
\hline 999281-36 & 35 & silt and clay, blocky & 13.41 & 1.74 & 5.83 & 3.08 & 1.92 & 0.16 & 1.91 & 0.17 & 71.15 & 0.63 & 16 & 2 & 40 & 57 & 1,200 & $<2$ & 200 \\
\hline 999281-38 & 37 & fine sand and silt & 13.04 & 1.33 & 5.22 & 3.10 & 1.73 & 0.15 & 2.02 & 0.15 & 72.71 & 0.56 & 16 & 2 & 75 & 52 & 1,100 & $<2$ & 200 \\
\hline $999281-40$ & 39 & fine sand and silt & 13.02 & 1.38 & 5.34 & 3.05 & 1.77 & 0.17 & 2.01 & 0.17 & 72.51 & 0.59 & 13 & 2 & 39 & 52 & 1,300 & $<2$ & 180 \\
\hline 999281-42 & 41 & fine sand and silt & 13.03 & 1.42 & 5.34 & 3.05 & 1.78 & 0.16 & 1.95 & 0.18 & 72.48 & 0.61 & 19 & 2 & 41 & 52 & 1,300 & $<2$ & 180 \\
\hline
\end{tabular}


Table 3. Core descriptions, depths, and geochemical data from core 97ABS300.

[Core descriptions from Fey and others (2000), table 5; ICP-AES data from table 9; sulfur and $x$-ray fluorescence data by contract laboratory]

\begin{tabular}{|c|c|c|c|c|c|c|c|c|c|c|c|c|c|c|c|c|c|c|c|c|}
\hline \multirow[b]{2}{*}{ Field sample no. } & \multirow{2}{*}{$\begin{array}{c}\text { Sample } \\
\text { interval } \\
\text { midpoint } \\
\text { depth } \\
\text { (cm) }\end{array}$} & \multirow[b]{2}{*}{ Subinterval sample description } & \multirow[b]{2}{*}{$\begin{array}{l}\text { Sulfur } \\
\text { wt. } \%\end{array}$} & \multicolumn{10}{|c|}{ X-ray fluorescence data } & \multicolumn{7}{|c|}{ ICP-AES data } \\
\hline & & & & $\begin{array}{l}\mathrm{Al}_{2} \mathrm{O}_{3} \\
\text { wt. \% }\end{array}$ & $\begin{array}{l}\mathrm{CaO} \\
\mathrm{wt} . \%\end{array}$ & $\begin{array}{l}\mathrm{Fe}_{2} \mathrm{O}_{3} \\
\text { wt. \% }\end{array}$ & $\begin{array}{c}\mathrm{K}_{2} \mathrm{O} \\
\mathrm{wt.} \%\end{array}$ & $\begin{array}{l}\mathrm{Mg0} \\
\mathrm{wt.} \%\end{array}$ & $\begin{array}{l}\mathrm{Mn0} \\
\mathrm{wt} . \%\end{array}$ & $\begin{array}{l}\mathrm{Na}_{2} \mathrm{O} \\
\text { wt. } \%\end{array}$ & $\begin{array}{l}\mathbf{P}_{2} \mathbf{0}_{5} \\
\text { wt. \% }\end{array}$ & $\begin{array}{r}\mathrm{SiO}_{2} \\
\mathrm{wt.} \%\end{array}$ & $\begin{array}{r}\mathrm{TiO}_{2} \\
\text { wt. } \%\end{array}$ & $\begin{array}{c}\text { Arsenic } \\
\mathrm{ppm}\end{array}$ & $\begin{array}{l}\text { Cadmium } \\
\text { ppm }\end{array}$ & $\begin{array}{c}\text { Copper } \\
\text { ppm }\end{array}$ & $\begin{array}{l}\text { Lead } \\
\text { ppm }\end{array}$ & $\begin{array}{l}\text { Manganese } \\
\text { ppm }\end{array}$ & $\begin{array}{c}\text { Silver } \\
\text { ppm }\end{array}$ & $\begin{array}{l}\text { Zinc } \\
\text { ppm }\end{array}$ \\
\hline $97 \mathrm{ABS} 300 \mathrm{~A}-\mathrm{a} 2$ & 6 & medium-brown silt, micaceous, soil, grass roots & 0.12 & 11.43 & 1.87 & 4.55 & 2.76 & 1.50 & 0.78 & 1.40 & 0.18 & 74.92 & 0.61 & 28 & 8 & 270 & 700 & 5,500 & 6 & 1,700 \\
\hline 97ABS300A-b2 & 21 & dark-brown fine sand and silt, roots & 1.44 & 10.30 & 3.74 & 3.51 & 2.29 & 1.18 & 2.42 & 0.88 & 0.19 & 74.96 & 0.52 & 25 & 22 & 300 & 980 & 14,000 & 10 & 3,200 \\
\hline 97ABS300A-c2 & 47 & $\begin{array}{l}\text { medium-brown fine sand and silt, mica, minor iron- } \\
\text { oxide staining }\end{array}$ & 0.41 & 9.44 & 2.12 & 3.76 & 1.95 & 1.04 & 2.93 & 0.94 & 0.18 & 77.19 & 0.44 & 34 & 4 & 450 & 2,800 & 19,000 & 21 & 700 \\
\hline 97ABS300A-d2 & 59 & $\begin{array}{l}\text { medium-brown and tan silt and clay, fine sand, } \\
\text { minor orange iron-oxidation staining, few root } \\
\text { twigs }\end{array}$ & 0.77 & 9.95 & 2.30 & 3.23 & 2.06 & 1.10 & 2.88 & 1.07 & 0.15 & 76.85 & 0.42 & 25 & 6 & 530 & 1,300 & 18,000 & 14 & 1,100 \\
\hline 97ABS300A-e1 & 67 & $\begin{array}{l}\text { tan fine sand and silt, moderate iron-oxide staining, } \\
\text { some root twigs }\end{array}$ & 0.44 & 8.72 & 2.06 & 3.43 & 1.78 & 0.97 & 3.03 & 0.89 & 0.16 & 78.57 & 0.39 & 29 & 3 & 350 & 2,200 & 19,000 & 19 & 650 \\
\hline 97ABS300A-f1 & 74 & tan and buff fine sand & 1.39 & 7.64 & 2.86 & 2.96 & 1.38 & 0.79 & 4.19 & 0.63 & 0.15 & 79.07 & 0.34 & 35 & 3 & 500 & 2,900 & 24,000 & 25 & 690 \\
\hline 97ABS300A-g1 & 77 & $\begin{array}{l}\text { dark and tan fine sand, one 1-cm-diameter root } \\
\text { fragment }\end{array}$ & 1.40 & 9.28 & 2.53 & 2.95 & 1.77 & 0.90 & 4.51 & 0.92 & 0.15 & 76.59 & 0.40 & 29 & 6 & 650 & 1,700 & 24,000 & 19 & 1,100 \\
\hline 97ABS300A-h1 & 81 & $\begin{array}{l}\text { dark and tan fine sand, a few 2-mm root twigs, pos- } \\
\text { sible clay in sample }\end{array}$ & 1.05 & 9.88 & 2.49 & 3.26 & 1.95 & 1.08 & 3.69 & 0.98 & 0.16 & 76.05 & 0.46 & 28 & 7 & 500 & 1,700 & 22,000 & 18 & 1,200 \\
\hline 97ABS300A-i3 & 93 & light-tan fine sand and silt, no rootlets & 1.64 & 9.45 & 2.98 & 2.81 & 1.74 & 1.00 & 4.72 & 0.77 & 0.16 & 75.98 & 0.40 & 29 & 7 & 530 & 2,000 & 26,000 & 19 & 1,700 \\
\hline 97ABS300A-j1 & 112 & $\begin{array}{l}\text { medium-brown fine sand, a little orange staining, } \\
\text { sparse rootlets }\end{array}$ & 0.84 & 9.39 & 2.39 & 2.45 & 1.77 & 0.96 & 4.51 & 0.89 & 0.16 & 77.07 & 0.41 & 26 & 10 & 510 & 1,900 & 26,000 & 19 & 1,300 \\
\hline 97ABS300A-k3 & 126 & $\begin{array}{l}\text { orange-buff silt, strong iron-oxide staining, root } \\
\text { twig to 8-mm diameter }\end{array}$ & 0.54 & 8.02 & 2.70 & 2.53 & 1.40 & 0.81 & 4.77 & 0.58 & 0.15 & 78.71 & 0.33 & 33 & 3 & 540 & 2,800 & 27,000 & 26 & 850 \\
\hline 97ABS300A-k5 & 133 & orange-buff silt & 1.13 & 7.33 & 4.32 & 2.00 & 1.22 & 0.70 & 5.38 & 0.44 & 0.14 & 78.17 & 0.29 & 37 & 4 & 630 & 4,400 & 28,000 & 32 & 770 \\
\hline 97ABS300A-m1 & 141 & $\begin{array}{l}\text { buff-tan and darker, fine sand and silt, little iron- } \\
\text { oxidation, mica }\end{array}$ & 0.80 & 8.63 & 2.15 & 2.57 & 1.75 & 0.86 & 3.42 & 0.94 & 0.14 & 79.17 & 0.37 & 26 & 8 & 600 & 1,600 & 23,000 & 16 & 1,100 \\
\hline 97ABS300A-n2 & 154 & buff-tan fine sand and silt, a few small twigs & 1.03 & 9.24 & 2.45 & 2.58 & 1.85 & 0.98 & 3.59 & 0.91 & 0.14 & 77.88 & 0.39 & 26 & 8 & 470 & 1,300 & 21,000 & 14 & 1,100 \\
\hline 97ABS300A-o2 & 181 & dark gray-brown silt, mafics, some root fibers & 0.07 & 11.57 & 1.47 & 3.77 & 2.51 & 1.34 & 2.35 & 1.40 & 0.18 & 74.87 & 0.54 & 23 & 8 & 440 & 1,100 & 15,000 & 10 & 1,200 \\
\hline 97ABS300A-p2 & 194 & gray-brown fine sand, sparse root fibers and twigs & 0.14 & 10.57 & 1.32 & 3.42 & 2.43 & 1.09 & 1.61 & 1.62 & 0.15 & 77.30 & 0.48 & 17 & 6 & 310 & 590 & 11,000 & 7 & 890 \\
\hline $97 \mathrm{ABS} 300 \mathrm{~A}-\mathrm{q} 2$ & 218 & $\begin{array}{l}\text { medium-brown fine sand and silt, micaceous, minor } \\
\text { iron-oxide staining, a few root fibers }\end{array}$ & 0.07 & 10.40 & 1.44 & 3.55 & 2.41 & 1.12 & 1.44 & 1.55 & 0.15 & 77.44 & 0.50 & 20 & 9 & 340 & 580 & 10,000 & 8 & 2,000 \\
\hline $97 \mathrm{ABS} 300 \mathrm{~A}-\mathrm{r} 2$ & 231 & tan-gray silt, some scattered iron-oxide staining & 0.10 & 11.62 & 1.85 & 4.12 & 2.61 & 1.55 & 1.38 & 1.29 & 0.19 & 74.81 & 0.59 & 26 & 13 & 410 & 1,200 & 9,000 & 8 & 3,700 \\
\hline
\end{tabular}


Table 4. Core descriptions, depths, and geochemical data from core 99929-1.

[Core descriptions from Fey and others (2000), table 2; ICP-AES data from table 6]

\begin{tabular}{|c|c|c|c|c|c|c|c|c|c|c|c|c|c|c|c|c|}
\hline \multirow[b]{2}{*}{$\begin{array}{c}\text { Field sample } \\
\text { no. }\end{array}$} & \multirow[b]{2}{*}{$\begin{array}{l}\text { Sample interval } \\
\text { midpoint depth } \\
\text { (cm) }\end{array}$} & \multirow[b]{2}{*}{ Subinterval sample description } & \multicolumn{14}{|c|}{ ICP-AES data } \\
\hline & & & $\begin{array}{c}\text { Aluminum } \\
\text { wt. } \%\end{array}$ & $\begin{array}{l}\text { Calcium } \\
\text { wt. } \%\end{array}$ & $\begin{array}{l}\text { Magnesium } \\
\text { wt. \% }\end{array}$ & $\begin{array}{l}\text { Iron } \\
\text { wt. \% }\end{array}$ & $\begin{array}{c}\text { Sodium } \\
\text { wt. } \%\end{array}$ & $\begin{array}{l}\text { Potassium } \\
\text { wt. \% }\end{array}$ & $\begin{array}{c}\text { Titanium } \\
\text { wt. \% }\end{array}$ & $\begin{array}{c}\text { Arsenic } \\
\text { ppm }\end{array}$ & $\begin{array}{c}\text { Cadmium } \\
\text { ppm }\end{array}$ & $\begin{array}{c}\text { Copper } \\
\text { ppm }\end{array}$ & $\begin{array}{l}\text { Lead } \\
\mathrm{ppm}\end{array}$ & $\begin{array}{l}\text { Manganese } \\
\text { ppm }\end{array}$ & $\begin{array}{l}\text { Silver } \\
\text { ppm }\end{array}$ & $\begin{array}{l}\text { Zinc } \\
\text { ppm }\end{array}$ \\
\hline $\begin{array}{l}999291-1 \\
\end{array}$ & 1 & light-brown silt & 2.8 & 0.09 & 0.23 & 30 & 0.26 & 0.63 & 0.09 & 14 & 8 & 10 & 8 & 68 & $<2$ & 39 \\
\hline 999291-2 & 2 & light-brown silt & 4.2 & 0.14 & 0.31 & 20 & 0.34 & 0.82 & 0.12 & $<10$ & 5 & 19 & 6 & 98 & $<2$ & 42 \\
\hline 999291-3 & 3 & light-brown silt & 3.0 & 0.15 & 0.24 & 30 & 0.24 & 0.61 & 0.09 & $<10$ & 9 & 4 & 9 & 71 & $<2$ & 30 \\
\hline 999291-4 & 4 & light-brown silt & 3.2 & 0.19 & 0.26 & 30 & 0.25 & 0.66 & 0.09 & 13 & 9 & 6 & 6 & 80 & $<2$ & 26 \\
\hline 999291-5 & 5 & light-brown silt, some orange coloration & 4.8 & 0.23 & 0.35 & 25 & 0.37 & 0.96 & 0.11 & 16 & 8 & 32 & 13 & 130 & $<2$ & 33 \\
\hline 999291-6 & 6 & light-brown silt, some orange coloration & 6.0 & 0.20 & 0.47 & 21 & 0.41 & 1.30 & 0.14 & 16 & 6 & 13 & 24 & 180 & $<2$ & 39 \\
\hline 999291-7 & 7 & light-brown silt, some orange coloration & 5.3 & 0.18 & 0.42 & 25 & 0.34 & 1.10 & 0.13 & 12 & 8 & 11 & 20 & 140 & $<2$ & 48 \\
\hline 999291-8 & 8 & light-brown silt, some orange coloration & 3.4 & 0.13 & 0.24 & 34 & 0.21 & 0.64 & 0.09 & 11 & 9 & 4 & 9 & 59 & $<2$ & 36 \\
\hline 999291-9 & 9 & light-brown silt, some orange coloration & 2.3 & 0.09 & 0.12 & 41 & 0.12 & 0.32 & 0.06 & $<10$ & 11 & $<1$ & $<4$ & 5 & $<2$ & 27 \\
\hline 999291-10 & 10 & light-brown silt, some orange coloration & 2.2 & 0.08 & 0.10 & 38 & 0.10 & 0.27 & 0.05 & $<10$ & 10 & 3 & $<4$ & 4 & $<2$ & 25 \\
\hline 999291-12 & 12 & light-brown silt, some orange coloration & 2.2 & 0.08 & 0.11 & 39 & 0.10 & 0.26 & 0.05 & $<10$ & 10 & 8 & $<4$ & $<4$ & $<2$ & 33 \\
\hline 999291-14 & 14 & light-brown silt, some orange coloration & 2.5 & 0.10 & 0.12 & 36 & 0.12 & 0.30 & 0.05 & $<10$ & 9 & $<1$ & $<4$ & 9 & $<2$ & 44 \\
\hline 999291-16 & 16 & light-brown silt, some orange coloration & 2.8 & 0.11 & 0.13 & 36 & 0.12 & 0.31 & 0.06 & $<10$ & 10 & 9 & $<4$ & 12 & $<2$ & 54 \\
\hline 999291-18 & 18 & light-brown silt, less orange & 2.4 & 0.13 & 0.13 & 32 & 0.13 & 0.33 & 0.06 & $<10$ & 8 & 12 & $<4$ & 15 & $<2$ & 62 \\
\hline 999291-20 & 20 & brown silt & 3.3 & 0.08 & 0.07 & 38 & 0.05 & 0.14 & 0.03 & $<10$ & 9 & $<1$ & $<4$ & $<4$ & $<2$ & 68 \\
\hline $999291-22$ & 22 & brown silt & 3.3 & 0.11 & 0.09 & 36 & 0.07 & 0.18 & 0.04 & $<10$ & 10 & 7 & $<4$ & $<4$ & $<2$ & 27 \\
\hline 999291-24 & 24 & brown with orange silt & 3.3 & 0.06 & 0.06 & 39 & 0.04 & 0.11 & 0.03 & $<10$ & 10 & 8 & $<4$ & $<4$ & $<2$ & 86 \\
\hline 999291-26 & 26 & brown and orange silt, iron-oxide clumps & 3.2 & 0.03 & 0.05 & 40 & 0.04 & 0.10 & 0.03 & $<10$ & 10 & 1 & $<4$ & $<4$ & $<2$ & 52 \\
\hline 999291-28 & 28 & orange-brown silt with some plant fibers & 4.1 & 0.02 & 0.04 & 39 & 0.02 & 0.06 & 0.02 & $<10$ & 11 & 3 & $<4$ & $<4$ & $<2$ & 49 \\
\hline 999291-30 & 30 & orange-brown silt with some plant fibers & 4.9 & 0.02 & 0.04 & 35 & 0.03 & 0.08 & 0.02 & $<10$ & 10 & 9 & $<4$ & $<4$ & $<2$ & 61 \\
\hline 999291-32 & 32 & tan-brown silt & 5.0 & 0.03 & 0.08 & 27 & 0.07 & 0.17 & 0.04 & 29 & 8 & $<1$ & $<4$ & $<4$ & $<2$ & 48 \\
\hline 999291-34 & 34 & orange-brown silt with grass fibers & 5.0 & 0.02 & 0.06 & 27 & 0.05 & 0.13 & 0.03 & $<10$ & 8 & 7 & $<4$ & $<4$ & $<2$ & 40 \\
\hline 999291-36 & 36 & brown-orange silt with grass fibers & 5.5 & 0.02 & 0.05 & 27 & 0.04 & 0.11 & 0.03 & $<10$ & 7 & $<1$ & $<4$ & $<4$ & $<2$ & 28 \\
\hline 999291-38 & 38 & brown-orange silt with grass fibers & 5.8 & 0.03 & 0.08 & 22 & 0.08 & 0.19 & 0.04 & $<10$ & 7 & 6 & $<4$ & 6 & $<2$ & 30 \\
\hline 999291-40 & 40 & tan-brown silt & 5.8 & 0.05 & 0.09 & 20 & 0.10 & 0.22 & 0.05 & $<10$ & 6 & 1 & $<4$ & 9 & $<2$ & 40 \\
\hline $999291-42$ & 42 & brown-gray silt and matted fibers & 5.8 & 0.06 & 0.10 & 17 & 0.11 & 0.23 & 0.05 & 10 & 5 & 4 & $<4$ & 14 & $<2$ & 36 \\
\hline 999291-44 & 44 & gray-brown silt & 5.3 & 0.07 & 0.11 & 12 & 0.13 & 0.26 & 0.06 & $<10$ & 3 & 13 & $<4$ & 19 & $<2$ & 36 \\
\hline 999291-46 & 46 & gray-brown silt & 4.2 & 0.07 & 0.10 & 8.4 & 0.10 & 0.23 & 0.05 & $<10$ & 3 & 10 & $<4$ & 25 & $<2$ & 29 \\
\hline 999291-48 & 48 & gray-brown silt & 3.9 & 0.07 & 0.10 & 6.6 & 0.11 & 0.23 & 0.04 & $<10$ & 3 & 12 & $<4$ & 22 & $<2$ & 26 \\
\hline 999291-50 & 50 & matted fibers & 4.3 & 0.08 & 0.12 & 5.3 & 0.12 & 0.25 & 0.04 & $<10$ & 3 & 12 & 5 & 33 & $<2$ & 31 \\
\hline 999291-52 & 52 & fine mix of fiber and silt & 4.3 & 0.10 & 0.12 & 5.1 & 0.13 & 0.26 & 0.04 & $<10$ & 3 & 12 & 4 & 33 & $<2$ & 39 \\
\hline 999291-54 & 54 & fine mix of fiber and silt & 4.3 & 0.09 & 0.15 & 8.9 & 0.16 & 0.38 & 0.06 & $<10$ & 3 & 13 & 5 & 40 & $<2$ & 38 \\
\hline
\end{tabular}


Table 5. Core descriptions, depths, and geochemical data from core 99929-2.

[Core descriptions from Fey and others (2000), table 2; ICP-AES data from table 6; sulfur and $\mathrm{x}$-ray fluorescence data by contract laboratory; --, no data]

\begin{tabular}{|c|c|c|c|c|c|c|c|c|c|c|c|c|c|c|c|c|c|c|c|c|}
\hline \multirow[b]{2}{*}{$\begin{array}{c}\text { Field sample } \\
\text { no. }\end{array}$} & \multirow{2}{*}{$\begin{array}{c}\text { Sample } \\
\text { interval } \\
\text { midpoint } \\
\text { depth } \\
\text { (cm) }\end{array}$} & \multirow[b]{2}{*}{ Subinterval sample description } & \multirow[b]{2}{*}{$\begin{array}{l}\text { Sulfur } \\
\text { wt. } \%\end{array}$} & \multicolumn{10}{|c|}{ X-ray fluorescence data } & \multicolumn{7}{|c|}{ ICP-AES data } \\
\hline & & & & $\begin{array}{l}\mathrm{Al}_{2} \mathrm{O}_{3} \\
\text { wt. \% }\end{array}$ & $\begin{array}{l}\mathrm{CaO} \\
\mathrm{wt.} \%\end{array}$ & $\begin{array}{l}\mathrm{Fe}_{2} \mathrm{O}_{3} \\
\mathrm{wt.} \%\end{array}$ & $\begin{array}{l}\mathrm{K}_{2} \mathbf{0} \\
\mathrm{wt.} \%\end{array}$ & $\begin{array}{l}\mathrm{MgO} \\
\text { wt. \% }\end{array}$ & $\begin{array}{l}\text { Mn0 } \\
\text { wt. \% }\end{array}$ & $\begin{array}{l}\mathrm{Na}_{2} \mathrm{O} \\
\text { wt. \% }\end{array}$ & $\begin{array}{l}\mathrm{P}_{2} \mathbf{O}_{5} \\
\text { wt. \% }\end{array}$ & $\begin{array}{l}\mathrm{SiO}_{2} \\
\text { wt. } \%\end{array}$ & $\begin{array}{r}\mathrm{TiO}_{2} \\
\text { wt. } \%\end{array}$ & $\begin{array}{c}\text { Arsenic } \\
\mathrm{ppm}\end{array}$ & $\begin{array}{c}\text { Cadmium } \\
\text { ppm }\end{array}$ & $\begin{array}{c}\text { Copper } \\
\text { ppm }\end{array}$ & $\begin{array}{l}\text { Lead } \\
\mathrm{ppm}\end{array}$ & $\begin{array}{c}\text { Manganese } \\
\text { ppm }\end{array}$ & $\begin{array}{c}\text { Silver } \\
\text { ppm }\end{array}$ & $\begin{array}{l}\text { Zinc } \\
\text { ppm }\end{array}$ \\
\hline 999292-2 & 2 & tan-gray silt, one root twig discarded & 4.42 & 9.07 & 1.56 & 13.18 & 1.91 & 1.15 & 0.03 & 0.41 & 0.73 & 71.56 & 0.41 & 30 & 9 & 150 & 210 & 170 & $<2$ & 2,200 \\
\hline $999292-3$ & 3 & tan-gray silt & 6.43 & 9.18 & 1.74 & 14.87 & 1.85 & 1.15 & 0.04 & 0.42 & 0.58 & 69.76 & 0.41 & 31 & 10 & 160 & 210 & 200 & $<2$ & 2,600 \\
\hline 999292-4 & 4 & tan-gray silt & 7.62 & 10.25 & 2.07 & 15.66 & 1.97 & 1.22 & 0.06 & 0.53 & 0.46 & 67.34 & 0.44 & 38 & 9 & 200 & 240 & 240 & $<2$ & 2,200 \\
\hline 999292-5 & 5 & tan-gray silt & 8.28 & 11.62 & 2.06 & 16.44 & 2.15 & 1.30 & 0.06 & 0.57 & 0.44 & 64.88 & 0.48 & 57 & 10 & 260 & 280 & 260 & $<2$ & 2,200 \\
\hline 999292-6 & 6 & tan-gray silt & 9.30 & 13.60 & 2.18 & 18.35 & 2.38 & 1.39 & 0.06 & 0.64 & 0.61 & 60.25 & 0.54 & 55 & 9 & 330 & 310 & 290 & $<2$ & 2,100 \\
\hline 999292-7 & 7 & tan-gray silt & 11.05 & 12.54 & 2.48 & 22.61 & 2.35 & 1.50 & 0.09 & 0.67 & 0.48 & 56.75 & 0.54 & 49 & 9 & 210 & 250 & 340 & $<2$ & 1,800 \\
\hline 999292-8 & 8 & tan-gray silt & -- & -- & -- & -- & -- & -- & -- & -- & -- & -- & -- & 42 & 8 & 130 & 270 & 300 & $<2$ & 1,500 \\
\hline $999292-9$ & 9 & tan-gray silt & 9.26 & 13.39 & 3.40 & 21.56 & 2.77 & 1.74 & 0.06 & 0.88 & 0.37 & 55.16 & 0.68 & 34 & 7 & 140 & 350 & 240 & $<2$ & 1,400 \\
\hline 999292-10 & 10 & tan-gray silt with some plant fibers & 9.56 & 13.31 & 3.53 & 22.78 & 2.75 & 1.73 & 0.07 & 0.89 & 0.38 & 53.89 & 0.66 & 48 & 7 & 140 & 350 & 230 & $<2$ & 1,400 \\
\hline $999292-12$ & 12 & tan-gray silt & 7.64 & 15.07 & 3.70 & 20.45 & 3.13 & 1.94 & 0.07 & 0.98 & 0.44 & 53.46 & 0.77 & 50 & 7 & 120 & 500 & 220 & $<2$ & 1,600 \\
\hline 999292-14 & 14 & matted fiber & -- & 18.04 & 2.81 & 10.39 & 3.50 & 2.14 & 0.06 & 1.34 & 0.38 & 60.55 & 0.80 & 36 & 5 & 120 & 700 & 290 & 2 & 950 \\
\hline $999292-16$ & 16 & matted fiber & -- & 22.99 & 2.92 & 5.60 & 2.78 & 1.86 & 0.06 & 1.39 & 0.28 & 61.33 & 0.79 & 26 & 4 & 97 & 540 & 240 & $<2$ & 910 \\
\hline 999292-18 & 18 & matted fiber & -- & 14.97 & 3.52 & 5.93 & 2.87 & 1.70 & 0.04 & 1.67 & 0.34 & 68.12 & 0.83 & 18 & 3 & 54 & 270 & 230 & $<2$ & 740 \\
\hline $999292-20$ & 20 & matted fiber & -- & 14.79 & 4.23 & 5.73 & 2.81 & 1.74 & 0.04 & 1.69 & 0.43 & 67.71 & 0.83 & 15 & $<2$ & 36 & 99 & 190 & $<2$ & 200 \\
\hline $999292-22$ & 22 & matted fiber & -- & 14.98 & 4.15 & 5.53 & 2.84 & 1.75 & 0.04 & 1.72 & 0.42 & 67.72 & 0.84 & 12 & $<2$ & 32 & 97 & 200 & $<2$ & 170 \\
\hline 999292-24 & 24 & silt and fine fiber & -- & 15.00 & 3.88 & 5.61 & 2.89 & 1.77 & 0.04 & 1.75 & 0.39 & 67.83 & 0.84 & 15 & $<2$ & 39 & 140 & 220 & $<2$ & 220 \\
\hline $999292-26$ & 26 & gray-brown silt and mats & -- & 14.77 & 4.07 & 6.38 & 2.82 & 1.73 & 0.04 & 1.69 & 0.43 & 67.22 & 0.83 & 14 & $<2$ & 34 & 120 & 210 & $<2$ & 210 \\
\hline $999292-28$ & 28 & gray-brown silt and mats & -- & 14.26 & 5.14 & 9.68 & 2.71 & 1.72 & 0.05 & 1.54 & 0.48 & 63.61 & 0.81 & 10 & $<2$ & 30 & 63 & 160 & $<2$ & 170 \\
\hline 999292-30 & 30 & clumps of matted fiber and silt & -- & 12.72 & 4.83 & 18.39 & 2.37 & 1.57 & 0.05 & 1.34 & 0.53 & 57.46 & 0.74 & 18 & 2 & 25 & 39 & 130 & $<2$ & 250 \\
\hline 999292-32 & 32 & gray silt & -- & 15.36 & 3.37 & 9.01 & 2.82 & 1.92 & 0.06 & 1.69 & 0.40 & 64.50 & 0.87 & 16 & 2 & 35 & 60 & 260 & $<2$ & 240 \\
\hline 999292-34 & 34 & gray and tan clumps of silt and fiber & -- & 16.21 & 2.83 & 10.38 & 2.90 & 2.08 & 0.06 & 1.61 & 0.37 & 62.71 & 0.87 & 20 & 3 & 40 & 78 & 300 & $<2$ & 200 \\
\hline 999292-36 & 36 & tan-brown silt, little fiber & 2.16 & 16.78 & 2.80 & 9.65 & 3.06 & 2.10 & 0.07 & 1.56 & 0.34 & 62.74 & 0.90 & 24 & 3 & 46 & 87 & 320 & $<2$ & 220 \\
\hline 999292-38 & 38 & gray-brown clumps of clay and silt and fiber & -- & 18.53 & 3.11 & 7.84 & 3.25 & 2.26 & 0.06 & 1.41 & 0.47 & 62.33 & 0.75 & 12 & 3 & 54 & 78 & 270 & $<2$ & 230 \\
\hline 999292-40 & 40 & medium-brown material & -- & 17.55 & 10.40 & 13.98 & 2.84 & 2.08 & 0.04 & 0.90 & 1.18 & 50.39 & 0.64 & $<10$ & $<2$ & 70 & 23 & 74 & $<2$ & 96 \\
\hline 999292-42 & 42 & medium-brown material & -- & 13.89 & 14.07 & 17.62 & 2.34 & 1.70 & 0.03 & 0.84 & 1.08 & 47.80 & 0.63 & $<10$ & 3 & 43 & 11 & 39 & $<2$ & 420 \\
\hline 999292-44 & 44 & medium-brown material & -- & 13.30 & 8.81 & 31.94 & 2.51 & 1.79 & 0.05 & 0.86 & 0.69 & 39.42 & 0.63 & 11 & 4 & 32 & 30 & 92 & $<2$ & 490 \\
\hline $999292-46$ & 46 & medium-brown material & -- & 13.47 & 6.07 & 22.59 & 2.47 & 1.65 & 0.05 & 0.99 & 0.66 & 51.42 & 0.64 & 19 & 4 & 69 & 120 & 130 & $<2$ & 570 \\
\hline
\end{tabular}


Table 6. Core descriptions, depths, and geochemical data from core 99929-3.

[Core descriptions from Fey and others (2000), table 2; ICP-AES data from table 6]

\begin{tabular}{|c|c|c|c|c|c|c|c|c|c|c|c|c|c|c|c|c|}
\hline \multirow[b]{2}{*}{$\begin{array}{c}\text { Field sample } \\
\text { no. }\end{array}$} & \multirow[b]{2}{*}{$\begin{array}{l}\text { Sample interval } \\
\text { midpoint depth } \\
\text { (cm) }\end{array}$} & \multirow[b]{2}{*}{ Subinterval sample description } & \multicolumn{14}{|c|}{ ICP-AES data } \\
\hline & & & $\begin{array}{c}\text { Aluminum } \\
\text { wt. } \%\end{array}$ & $\begin{array}{l}\text { Calcium } \\
\text { wt. } \%\end{array}$ & $\begin{array}{l}\text { Magnesium } \\
\text { wt. } \%\end{array}$ & $\begin{array}{l}\text { Iron } \\
\text { wt. \% }\end{array}$ & $\begin{array}{l}\text { Sodium } \\
\text { wt. } \%\end{array}$ & $\begin{array}{l}\text { Potassium } \\
\text { wt. } \%\end{array}$ & $\begin{array}{l}\text { Titanium } \\
\text { wt. } \%\end{array}$ & $\begin{array}{c}\text { Arsenic } \\
\text { ppm }\end{array}$ & $\begin{array}{c}\text { Cadmium } \\
\text { ppm }\end{array}$ & $\begin{array}{c}\text { Copper } \\
\text { ppm }\end{array}$ & $\begin{array}{l}\text { Lead } \\
\mathrm{ppm}\end{array}$ & $\begin{array}{c}\text { Manganese } \\
\text { ppm }\end{array}$ & $\begin{array}{l}\text { Silver } \\
\text { ppm }\end{array}$ & $\begin{array}{l}\text { Zinc } \\
\text { ppm }\end{array}$ \\
\hline 999293-2 & 2 & orange clayey silt & 3.8 & 0.09 & 0.34 & 24.0 & 0.34 & 0.86 & 0.10 & 15 & 6 & 21 & 15 & 120 & $<2$ & 51 \\
\hline 999293-3 & 3 & orange clayey silt & 5.0 & 0.11 & 0.42 & 21.0 & 0.48 & 1.20 & 0.14 & 16 & 7 & 20 & 22 & 160 & $<2$ & 44 \\
\hline 999293-4 & 4 & orange clayey silt & 7.0 & 0.14 & 0.56 & 13.0 & 0.60 & 1.60 & 0.16 & 14 & 4 & 29 & 38 & 220 & $<2$ & 43 \\
\hline 999293-5 & 5 & tan-brown clayey silt & 7.6 & 0.22 & 0.69 & 5.3 & 0.80 & 1.80 & 0.19 & $<10$ & 2 & 28 & 53 & 320 & $<2$ & 54 \\
\hline 999293-6 & 6 & orange-brown clayey silt & 6.0 & 0.19 & 0.47 & 15.0 & 0.56 & 1.30 & 0.16 & 22 & 4 & 20 & 30 & 190 & $<2$ & 78 \\
\hline 999293-7 & 7 & tan-brown clayey silt & 5.0 & 0.16 & 0.63 & 6.9 & 0.73 & 1.20 & 0.19 & 16 & 2 & 22 & 28 & 310 & $<2$ & 86 \\
\hline 999293-8 & 8 & tan-brown clayey silt & 4.5 & 0.16 & 0.42 & 5.9 & 0.43 & 1.10 & 0.16 & 10 & 2 & 26 & 23 & 130 & $<2$ & 48 \\
\hline 999293-9 & 9 & tan-orange clayey silt & 4.5 & 0.15 & 0.42 & 10.0 & 0.42 & 1.10 & 0.16 & 15 & 3 & 20 & 21 & 120 & $<2$ & 66 \\
\hline 999293-10 & 10 & orange silt & 3.6 & 0.14 & 0.40 & 19.0 & 0.39 & 0.99 & 0.14 & $<10$ & 5 & 32 & 18 & 110 & $<2$ & 39 \\
\hline 999293-12 & 12 & orange silt & 2.2 & 0.09 & 0.23 & 29.0 & 0.24 & 0.61 & 0.10 & $<10$ & 8 & 8 & $<4$ & 48 & $<2$ & 28 \\
\hline 999293-14 & 14 & orange silt & 2.5 & 0.12 & 0.25 & 20.0 & 0.28 & 0.71 & 0.12 & $<10$ & 5 & 11 & $<4$ & 48 & $<2$ & 20 \\
\hline 999293-16 & 16 & $\tan$ silt & 2.9 & 0.14 & 0.28 & 5.5 & 0.37 & 0.92 & 0.15 & $<10$ & $<2$ & 17 & 7 & 56 & $<2$ & 16 \\
\hline 999293-18 & 18 & $\tan$ silt & 2.6 & 0.12 & 0.24 & 3.5 & 0.33 & 0.82 & 0.14 & $<10$ & $<2$ & 12 & 6 & 49 & $<2$ & 13 \\
\hline 999293-20 & 20 & light-tan silt & 2.2 & 0.11 & 0.20 & 3.0 & 0.29 & 0.68 & 0.11 & $<10$ & $<2$ & 12 & 4 & 42 & $<2$ & 12 \\
\hline 999293-22 & 22 & tan-gray silt & 2.6 & 0.14 & 0.21 & 3.0 & 0.34 & 0.83 & 0.14 & $<10$ & $<2$ & 19 & 8 & 45 & $<2$ & 14 \\
\hline 999293-24 & 24 & light-brown silt & 3.0 & 0.20 & 0.23 & 5.9 & 0.25 & 0.75 & 0.14 & $<10$ & 2 & 16 & 6 & 51 & $<2$ & 40 \\
\hline 999293-26 & 26 & light-brown silt, some fibrous material & 2.7 & 0.23 & 0.19 & 5.7 & 0.19 & 0.59 & 0.13 & $<10$ & 2 & 14 & 5 & 56 & $<2$ & 81 \\
\hline 999293-28 & 28 & medium-brown silt & 2.5 & 0.25 & 0.17 & 4.8 & 0.18 & 0.51 & 0.10 & $<10$ & 2 & 13 & 5 & 56 & $<2$ & 120 \\
\hline 999293-30 & 30 & medium-brown silt and fiber & 2.3 & 0.24 & 0.14 & 6.7 & 0.14 & 0.39 & 0.08 & 21 & 3 & 16 & 14 & 58 & $<2$ & 100 \\
\hline 999293-32 & 32 & medium-brown silt and fiber & 3.0 & 0.37 & 0.20 & 6.1 & 0.29 & 0.60 & 0.10 & 20 & 3 & 17 & 18 & 93 & $<2$ & 160 \\
\hline 999293-34 & 34 & medium-brown silt and much fiber & 3.9 & 0.44 & 0.25 & 13.0 & 0.31 & 0.70 & 0.12 & 44 & 6 & 21 & 24 & 130 & $<2$ & 370 \\
\hline 999293-36 & 36 & brown silt & 2.7 & 0.26 & 0.15 & 14.0 & 0.20 & 0.44 & 0.08 & 32 & 4 & 16 & 16 & 97 & $<2$ & 170 \\
\hline 999293-38 & 38 & blocky silt, clay and fibrous clumps & 5.0 & 0.50 & 0.32 & 6.2 & 0.50 & 0.93 & 0.15 & 18 & 3 & 24 & 26 & 150 & $<2$ & 120 \\
\hline 999293-40 & 40 & medium-brown silt and fine organic fibers & 4.9 & 0.29 & 0.14 & 2.7 & 0.15 & 0.35 & 0.06 & 15 & $<2$ & 31 & 24 & 51 & $<2$ & 92 \\
\hline 999293-42 & 42 & mostly pressed fiber & 4.4 & 0.24 & 0.08 & 2.2 & 0.08 & 0.19 & 0.04 & $<10$ & $<2$ & 20 & 13 & 35 & $<2$ & 70 \\
\hline 999293-44 & 44 & mostly pressed fiber & 3.3 & 0.18 & 0.07 & 9.3 & 0.06 & 0.15 & 0.03 & $<10$ & 4 & 9 & 6 & 40 & $<2$ & 54 \\
\hline 999293-46 & 46 & mostly pressed fiber & 3.9 & 0.17 & 0.10 & 6.6 & 0.06 & 0.21 & 0.04 & $<10$ & 2 & 10 & 4 & 35 & $<2$ & 33 \\
\hline 999293-48 & 48 & mostly pressed fiber & 4.6 & 0.18 & 0.09 & 3.5 & 0.07 & 0.20 & 0.04 & $<10$ & 2 & 13 & 6 & 31 & $<2$ & 54 \\
\hline 999293-50 & 50 & mostly pressed fiber & 3.5 & 0.14 & 0.07 & 7.6 & 0.08 & 0.19 & 0.04 & $<10$ & 3 & 11 & $<4$ & 36 & $<2$ & 51 \\
\hline 999293-52 & 52 & mostly pressed fiber & 2.3 & 0.09 & 0.06 & 4.1 & 0.07 & 0.18 & 0.03 & $<10$ & $<2$ & 4 & $<4$ & 26 & $<2$ & 32 \\
\hline 999293-54 & 54 & mostly pressed fiber & 3.3 & 0.14 & 0.08 & 9.0 & 0.10 & 0.23 & 0.04 & $<10$ & 3 & 7 & $<4$ & 39 & $<2$ & 36 \\
\hline 999293-56 & 56 & mostly pressed fiber & 3.6 & 0.13 & 0.10 & 6.6 & 0.07 & 0.24 & 0.04 & $<10$ & 3 & 12 & $<4$ & 36 & $<2$ & 44 \\
\hline 999293-58 & 58 & mostly pressed fiber & 3.4 & 0.13 & 0.09 & 7.8 & 0.09 & 0.24 & 0.04 & $<10$ & 3 & 13 & $<4$ & 34 & $<2$ & 46 \\
\hline 999293-60 & 60 & mostly pressed fiber & 3.8 & 0.15 & 0.13 & 5.5 & 0.15 & 0.34 & 0.06 & $<10$ & 2 & 15 & 7 & 45 & $<2$ & 58 \\
\hline 999293-62 & 62 & mostly pressed fiber & 4.2 & 0.15 & 0.18 & 4.4 & 0.18 & 0.46 & 0.08 & 10 & $<2$ & 14 & 13 & 56 & $<2$ & 81 \\
\hline 999293-65 & 65 & mostly pressed fiber & 4.3 & 0.21 & 0.22 & 4.0 & 0.24 & 0.54 & 0.10 & $<10$ & $<2$ & 25 & 21 & 75 & $<2$ & 72 \\
\hline
\end{tabular}




\section{Radionuclide Analysis}

${ }^{210} \mathrm{~Pb}$ activity was measured by alpha spectroscopy. The ${ }^{210} \mathrm{~Pb}$ (half-life $=22.3 \mathrm{yr}$ ) alpha method is based on determining the activity of polonium-210 $\left({ }^{210} \mathrm{Po}\right)$, which is assumed to be in secular equilibrium with its parent ${ }^{210} \mathrm{~Pb}$. The analytical method exploits the ability of polonium to auto-plate onto silver planchets, which facilitates the alpha counting (Flynn, 1968). Briefly, $5 \mathrm{~g}$ of sediment was hand-ground in a mortar and pestle and fired in a muffle furnace at $550^{\circ} \mathrm{C}$ for 6 hours to determine loss-on-ignition (LOI). The percent ash content (determined from the LOI) of samples containing organic matter (peat) is provided for samples that were ashed prior to analysis. The fired material was transferred to a glass beaker, ${ }^{210}$ Po was acid-leached from the sediment, and a known quantity of the tracer ${ }^{209} \mathrm{Po}$ was added to the solution prior to auto-plating the polonium isotopes onto silver planchets. The planchets were counted in low-level alpha spectrometers coupled to a pulse-height analyzer.

Gamma spectroscopy was used to determine the beryllium-7 $\left({ }^{7} \mathrm{Be}\right)$ (half-life $\left.=53 \mathrm{~d}\right)$, cesium-137 $\left({ }^{137} \mathrm{Cs}\right)$ (half-life $=30.2 \mathrm{yr})$, and radium-226 $\left({ }^{226} \mathrm{Ra}\right)$ (half-life $\left.=1,600 \mathrm{yr}\right)$ activities in each sample (Cutshall and others, 1983). A 50-g aliquot of dried and ground sample was sealed in a plastic counting jar. Sealed samples were stored for at least 20 days to allow ${ }^{222} \mathrm{Ra}$ and ${ }^{214} \mathrm{~Pb}$ activity to come into equilibrium with their parent isotope $\left({ }^{226} \mathrm{Ra}\right)$. Samples were counted by a germanium detector $\left(2,000-\mathrm{mm}^{2}\right.$ area) for low-energy gamma rays, and data were collected using a 4,096 channel multi-channel analyzer. Samples were typically counted for 24 hours (depending on sample size) or until counting errors were less than 5 percent. The gamma system was calibrated using a NIST-traceable multi-line radioisotope standard in a soil matrix with the same counting geometry as the samples.
Specific activity of ${ }^{226} \mathrm{Ra}$ was determined from counts associated with the photo-peaks of ${ }^{214} \mathrm{~Pb}$ (295 and $351 \mathrm{kev}$ ) and ${ }^{214} \mathrm{Bi}$ (bismuth-214) (609 kev). Activities calculated from the three peaks were combined to yield a weighted-mean reported value and standard deviation. The gamma-ray counting system was calibrated and frequently checked by counting radioisotope standards prepared by doping portions of Florida Bay sediment with precisely known amounts of a radium standard solution (Robbins and others, 2000). This solution has a 0.4 percent uncertainty in activity at the 99 percent confidence level, which is due to random counting errors, and an additional 0.8 percent uncertainty due to assessable systematic counting errors. Reported standard deviations in ${ }^{226}$ Ra activity, including random errors associated with detector calibration, were typically 5-7 percent. The limit of detection was about $0.1 \mathrm{dpm} / \mathrm{g}$ (disintegrations per minute/gram) for this sample matrix. ${ }^{7} \mathrm{Be}$ and ${ }^{137} \mathrm{Cs}$ activities were determined by counting the $477 \mathrm{kev}$ and $661.6 \mathrm{kev}$ gamma ray peaks, respectively. All activities were decay corrected to the date of sample collection. The reported errors are the statistical counting errors at the 95 percent confidence interval. Total analytical errors are about \pm 6.5 percent based on replicate sample analyses. Analytical data are reported in tables 7-10.

Calculations of the ${ }^{210} \mathrm{~Pb}$ and ${ }^{137} \mathrm{Cs}$ flux $\left(\mathrm{dpm} / \mathrm{cm}^{2} / \mathrm{yr}\right)$ and inventories $\left(\mathrm{dpm} / \mathrm{cm}^{2}\right)$ are also included in tables $7-10$. The inventory of ${ }^{210} \mathrm{~Pb}$ was determined over the last $150 \mathrm{yr}$ in the dated portions of the cores and the flux estimated as that required to produce the observed inventory when corrected for decay. For ${ }^{137} \mathrm{Cs}$, the inventory is the sum over intervals where ${ }^{137} \mathrm{Cs}$ was detected, and the flux is the inventory divided by the number of years during which ${ }^{137} \mathrm{Cs}$ was observed. 
Table 7. Sample depths, loss-on-ignition, radionuclide activity, and inventory data, and ${ }^{210} \mathrm{~Pb}$ age calculations for core $99928-1$.

[Flux and inventory calculations completed by C.A. Rice; analytical data by M.E. Marot; --, not analyzed; **, not detected; dpm, disintegrations per minute]

\begin{tabular}{|c|c|c|c|c|c|c|c|c|c|c|c|c|c|c|c|}
\hline $\begin{array}{l}\text { Depth } \\
\text { (cm) }\end{array}$ & $\begin{array}{l}\text { Mean } \\
\text { depth } \\
(\mathrm{cm})\end{array}$ & $\begin{array}{l}\text { Dry bulk } \\
\text { density } \\
\left(\mathrm{g} / \mathrm{cm}^{3}\right)\end{array}$ & $\begin{array}{c}\text { Cumulative } \\
\text { sediment } \\
\text { weight } \\
\left(\mathbf{g} / \mathrm{cm}^{2}\right)\end{array}$ & $\begin{array}{c}\text { Loss-on- } \\
\text { ignition } \\
\text { (percent } \\
\text { dry wt.) }\end{array}$ & $\begin{array}{l}\text { Total }{ }^{210} \mathrm{~Pb} \\
\text { activity } \\
\text { (dpm/g) }\end{array}$ & $\begin{array}{l}\text { Total }{ }^{210} \mathrm{~Pb} \\
\text { activity error } \\
( \pm \mathrm{dpm} / \mathrm{g})\end{array}$ & $\begin{array}{c}\text { Excess }{ }^{210} \mathrm{~Pb} \\
\text { activity } \\
(\mathrm{dpm} / \mathrm{g})^{1}\end{array}$ & $\begin{array}{l}\text { Model } \\
\text { date }\end{array}$ & $\begin{array}{l}\text { Excess }{ }^{210} \mathrm{~Pb} \\
\text { inventory } \\
\left(\mathrm{dpm} / \mathrm{cm}^{2}\right)^{2}\end{array}$ & $\begin{array}{c}{ }^{226} \text { Ra activity } \\
(\mathrm{dpm} / \mathrm{g})\end{array}$ & $\begin{array}{l}{ }^{226} \mathrm{Ra} \text { activity } \\
\text { error }( \pm d p m / g)\end{array}$ & $\begin{array}{c}{ }^{137} \text { Cs activity } \\
(\mathrm{dpm} / \mathrm{g})\end{array}$ & $\begin{array}{c}{ }^{137} \text { Cs activity } \\
\text { error } \\
( \pm \mathrm{dpm} / \mathrm{g})\end{array}$ & $\begin{array}{c}{ }^{234} \mathrm{Th} \text { activity } \\
\text { (dpm/g) }\end{array}$ & $\begin{array}{c}{ }^{234} \mathrm{Th} \text { activity } \\
\quad \text { error } \\
( \pm \mathrm{dpm} / \mathrm{g})\end{array}$ \\
\hline $0-1$ & 0.5 & 0.273 & 0.273 & 20.20 & 12.68 & 0.17 & 11.127 & 1997 & 3.035 & 2.81 & 0.26 & 0.50 & 0.08 & 6.10 & 0.46 \\
\hline $1-2$ & 1.5 & 0.469 & 0.742 & 13.40 & 8.66 & 0.20 & 7.108 & 1994 & 3.336 & -- & -- & -- & -- & -- & -- \\
\hline $2-3$ & 2.5 & 0.610 & 1.352 & 10.80 & 6.14 & 0.08 & 4.590 & 1989 & 2.801 & -- & -- & -- & -- & -- & -- \\
\hline 3-4 & 3.5 & 0.863 & 2.215 & 9.00 & 4.93 & 0.07 & 3.381 & 1983 & 2.918 & 2.81 & 0.20 & 0.67 & 0.07 & 4.67 & 0.38 \\
\hline $4-5$ & 4.5 & 0.828 & 3.043 & 10.80 & 6.74 & 0.08 & 5.194 & 1976 & 4.301 & -- & -- & -- & -- & -- & -- \\
\hline $5-6$ & 5.5 & 0.875 & 3.918 & 11.20 & 5.89 & 0.08 & 4.337 & 1970 & 3.793 & -- & -- & -- & -- & -- & -- \\
\hline 6-7 & 6.5 & 0.741 & 4.659 & 9.00 & 3.84 & 0.06 & 2.291 & 1965 & 1.697 & 2.93 & 0.20 & 0.81 & 0.08 & 3.97 & 0.36 \\
\hline $7-8$ & 7.5 & 0.877 & 5.536 & 11.20 & 5.40 & 0.13 & 3.850 & 1958 & 3.376 & -- & -- & -- & -- & -- & -- \\
\hline 8-9 & 8.5 & 0.763 & 6.299 & 9.40 & 4.41 & 0.06 & 2.865 & 1952 & 2.187 & -- & -- & -- & -- & -- & -- \\
\hline $9-10$ & 9.5 & 1.063 & 7.363 & 9.40 & 3.53 & 0.05 & 1.982 & 1945 & 2.108 & 2.71 & 0.21 & 0.19 & 0.05 & 3.76 & 0.38 \\
\hline $10-12$ & 11.0 & 0.903 & 9.168 & 7.60 & 3.26 & 0.05 & 1.712 & 1931 & 3.091 & -- & -- & -- & -- & -- & -- \\
\hline $12-14$ & 13.0 & 1.090 & 11.348 & 7.60 & 2.21 & 0.04 & 0.665 & 1915 & 1.450 & -- & -- & -- & -- & -- & -- \\
\hline $14-16$ & 15.0 & 1.104 & 13.556 & 4.40 & 1.68 & 0.03 & 0.132 & 1899 & 0.291 & 2.52 & 0.19 & $* *$ & -- & 2.49 & 0.31 \\
\hline $16-18$ & 17.0 & 1.009 & 15.574 & 4.00 & 1.91 & 0.04 & 0.357 & 1884 & 0.720 & -- & -- & -- & -- & -- & -- \\
\hline $18-20$ & 19.0 & 1.030 & 17.634 & 4.20 & 1.51 & 0.03 & -0.045 & 1868 & 0.000 & -- & -- & -- & -- & -- & -- \\
\hline $20-22$ & 21.0 & 1.051 & 19.736 & 4.00 & 1.76 & 0.03 & 0.213 & 1853 & 0.447 & 2.67 & 0.20 & $* *$ & -- & 2.65 & 0.35 \\
\hline $22-24$ & 23.0 & 1.142 & 22.021 & 3.40 & 1.42 & 0.03 & -0.128 & 1836 & 0.000 & -- & -- & -- & -- & -- & -- \\
\hline $24-26$ & 25.0 & 1.183 & 24.387 & 2.80 & 1.52 & 0.03 & -0.029 & 1818 & 0.000 & -- & -- & -- & -- & -- & -- \\
\hline $26-28$ & 27.0 & 1.170 & 26.726 & 2.60 & 1.41 & 0.03 & -0.144 & 1801 & 0.000 & 2.45 & 0.19 & $* *$ & -- & 2.20 & 0.35 \\
\hline $28-30$ & 29.0 & 1.056 & 28.839 & 3.00 & 1.68 & 0.03 & 0.129 & 1786 & 0.272 & -- & -- & -- & -- & -- & -- \\
\hline $30-32$ & 31.0 & 1.130 & 31.098 & 3.20 & 1.71 & 0.04 & 0.161 & 1769 & 0.364 & -- & -- & -- & -- & -- & -- \\
\hline $32-34$ & 33.0 & 1.145 & 33.387 & 3.20 & 1.66 & 0.03 & 0.113 & 1752 & 0.259 & 2.61 & 0.18 & $* *$ & -- & 2.40 & 0.32 \\
\hline 34-36 & 35.0 & 1.106 & 35.600 & 2.20 & 1.37 & 0.03 & -0.176 & & & -- & -- & -- & -- & -- & -- \\
\hline $36-38$ & 37.0 & 1.217 & 38.033 & 2.40 & 1.32 & 0.03 & -0.226 & & & -- & -- & -- & -- & -- & -- \\
\hline $38-40$ & 39.0 & 1.177 & 40.386 & 3.20 & 1.35 & 0.03 & -0.196 & & & 2.26 & 0.15 & $* *$ & -- & 1.98 & 0.28 \\
\hline Bottom & 41.0 & 1.155 & 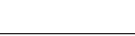 & 2.80 & 1.42 & 0.03 & & & & -- & -- & -- & -- & -- & -- \\
\hline
\end{tabular}

${ }^{2}$ Excess ${ }^{210} \mathrm{~Pb}$ Inventory $=35.55 \mathrm{dpm} / \mathrm{cm}^{2}$ (from top of core down to core interval $20-22 \mathrm{~cm}$, that is 150 years). 
Table 8. Sample depths, loss-on-ignition data, radionuclide activity, and inventory data for core 99929-1.

[Flux and inventory calculations completed by C.A. Rice; analytical data by M.E. Marot; --, not analyzed; **, not detected; $T$ r, too low to quantify; dpm, disintegrations per minute]

\begin{tabular}{|c|c|c|c|c|c|c|c|c|c|c|c|c|c|c|c|}
\hline $\begin{array}{l}\text { Depth } \\
\text { (cm) }\end{array}$ & $\begin{array}{l}\text { Mean } \\
\text { depth } \\
\text { (cm) }\end{array}$ & $\begin{array}{l}\text { Dry bulk } \\
\text { density } \\
\left(\mathbf{g} / \mathrm{cm}^{3}\right)\end{array}$ & $\begin{array}{c}\text { Cumulative } \\
\text { sediment weight } \\
\left(\mathbf{g} / \mathrm{cm}^{2}\right)\end{array}$ & $\begin{array}{c}\text { Loss-on- } \\
\text { ignition } \\
\text { (percent dry } \\
\text { wt.) }\end{array}$ & $\begin{array}{l}\text { Total }{ }^{20} \mathrm{~Pb} \\
\text { activity } \\
\text { (dpm/g) }\end{array}$ & $\begin{array}{l}\text { Total }{ }^{210} \mathrm{~Pb} \\
\text { activity error } \\
( \pm \mathrm{dpm} / \mathrm{g})\end{array}$ & $\begin{array}{l}\text { Excess }{ }^{210} \mathrm{~Pb} \\
\text { activity }(\mathrm{dpm} / \mathrm{g})\end{array}$ & $\begin{array}{l}\text { Excess }{ }^{210} \mathrm{~Pb} \\
\text { inventory } \\
\left(\mathrm{dpm} / \mathrm{cm}^{2}\right)^{1}\end{array}$ & $\begin{array}{l}{ }^{226} \mathrm{Ra} \\
\text { activity } \\
\text { (dpm/g) }\end{array}$ & $\begin{array}{c}{ }^{226} \mathrm{Ra} \\
\text { activity error } \\
( \pm \mathrm{dpm} / \mathrm{g})\end{array}$ & $\begin{array}{c}{ }^{137} \mathrm{Cs} \\
\text { activity } \\
\text { (dpm/g) }\end{array}$ & $\begin{array}{l}{ }^{137} \mathrm{Cs} \\
\text { activity error } \\
( \pm \mathrm{dpm} / \mathrm{g})\end{array}$ & $\begin{array}{c}{ }^{137} \mathrm{Cs} \\
\text { inventory } \\
\left(\mathrm{dpm} / \mathrm{cm}^{2}\right)^{2}\end{array}$ & $\begin{array}{l}{ }^{234} \mathrm{Th} \\
\text { activity } \\
\text { (dpm/g) }\end{array}$ & $\begin{array}{c}{ }^{234} \mathrm{Th} \\
\text { activity error } \\
( \pm \mathrm{dpm} / \mathrm{g})\end{array}$ \\
\hline$\overline{0-1}$ & 0.5 & 0.45 & 0.45 & 17.80 & 5.92 & 0.12 & 5.06 & 2.29 & 0.86 & 0.09 & 1.07 & 0.07 & 0.48 & 0.55 & 0.16 \\
\hline $1-2$ & 1.5 & 0.47 & 0.92 & 19.60 & 7.13 & 0.01 & 5.95 & 2.77 & 1.18 & 0.10 & 1.81 & 0.10 & 0.84 & 0.88 & 0.22 \\
\hline $2-3$ & 2.5 & 0.51 & 1.43 & 18.60 & 2.21 & 0.05 & 1.47 & 0.74 & 0.74 & 0.10 & 1.48 & 0.10 & 0.75 & $\operatorname{Tr}$ & -- \\
\hline $3-4$ & 3.5 & 0.45 & 1.87 & 18.80 & 2.02 & 0.02 & 1.41 & 0.63 & 0.61 & 0.08 & 0.67 & 0.06 & 0.30 & 0.59 & 0.15 \\
\hline $4-5$ & 4.5 & 0.45 & 2.32 & 17.00 & 2.40 & 0.02 & 1.09 & 0.49 & 1.31 & 0.15 & 0.47 & 0.08 & 0.21 & 1.16 & 0.33 \\
\hline $5-6$ & 5.5 & 0.45 & 2.77 & 13.80 & 2.78 & 0.02 & 1.17 & 0.53 & 1.61 & 0.12 & 0.22 & 0.05 & 0.10 & 0.91 & 0.26 \\
\hline 6-7 & 6.5 & 0.48 & 3.25 & 12.80 & 2.23 & 0.03 & 0.88 & 0.42 & 1.35 & 0.13 & 0.09 & 0.04 & 0.04 & $\mathrm{Tr}$ & -- \\
\hline $7-8$ & 7.5 & 0.66 & 3.90 & 13.00 & 1.40 & 0.02 & 0.67 & 0.44 & 0.73 & 0.07 & $* *$ & -- & & 0.50 & 0.13 \\
\hline 8-9 & 8.5 & 0.61 & 4.51 & 13.20 & 1.17 & 0.03 & 0.69 & 0.42 & 0.49 & 0.07 & $* *$ & -- & & $\mathrm{Tr}$ & -- \\
\hline $9-10$ & 9.5 & 0.61 & 5.12 & 13.00 & 0.89 & 0.03 & 0.44 & 0.27 & 0.45 & 0.07 & $* *$ & -- & & $\operatorname{Tr}$ & -- \\
\hline $10-12$ & 11 & 0.49 & 6.09 & 12.60 & 0.54 & 0.03 & 0.22 & 0.22 & 0.32 & 0.05 & $* *$ & -- & & $\operatorname{Tr}$ & -- \\
\hline $12-14$ & 13 & 0.40 & 6.89 & 12.60 & 0.64 & -- & 0.29 & 0.23 & 0.35 & 0.05 & $* *$ & -- & & $\operatorname{Tr}$ & -- \\
\hline $14-16$ & 15 & 0.38 & 7.65 & 12.20 & 0.64 & -- & 0.18 & 0.14 & 0.46 & 0.05 & $* *$ & -- & & $\mathrm{Tr}$ & -- \\
\hline 16-18 & 17 & 0.37 & 8.39 & 13.20 & 0.74 & 0.03 & 0.37 & 0.28 & 0.37 & 0.05 & $* *$ & -- & & 0.36 & 0.10 \\
\hline $18-20$ & 19 & 0.51 & 9.40 & 15.80 & 0.43 & 0.03 & 0.23 & 0.23 & 0.21 & 0.04 & $* *$ & -- & & 0.17 & 0.07 \\
\hline $20-22$ & 21 & 0.43 & 10.26 & 15.60 & 0.48 & 0.04 & & & -- & -- & -- & -- & & .- & -- \\
\hline $22-24$ & 23 & 0.50 & 11.26 & 14.00 & 0.33 & 0.09 & & & 0.18 & 0.04 & $* *$ & -- & & 0.18 & 0.08 \\
\hline $24-26$ & 25 & 0.52 & 12.31 & 13.00 & 0.38 & 0.07 & & & -- & -- & -- & -- & & -- & -- \\
\hline $26-28$ & 27 & 0.44 & 13.20 & 13.40 & 0.34 & 0.06 & & & 0.16 & 0.04 & $* *$ & -- & & $\operatorname{Tr}$ & -- \\
\hline $28-30$ & 29 & 0.42 & 14.04 & 14.40 & 0.32 & 0.05 & & & -- & -- & -- & -- & & -- & -- \\
\hline $30-32$ & 31 & 0.42 & 14.87 & 18.40 & 0.45 & 0.04 & & & 0.29 & 0.05 & $* *$ & -- & & 0.28 & 0.10 \\
\hline $32-34$ & 33 & 0.41 & 15.69 & 18.80 & 0.40 & 0.04 & & & -- & -- & -- & -- & & -- & -- \\
\hline $34-36$ & 35 & 0.45 & 16.60 & 18.20 & 0.24 & 0.04 & & & 0.12 & 0.03 & $* *$ & -- & & $\operatorname{Tr}$ & -- \\
\hline $36-38$ & 37 & 0.47 & 17.53 & 20.00 & 0.27 & 0.03 & & & -- & -- & -- & -- & & -- & -- \\
\hline $38-40$ & 39 & 0.48 & 18.49 & 21.20 & 0.30 & 0.06 & & & 0.20 & 0.04 & $* *$ & -- & & 0.23 & 0.10 \\
\hline $40-42$ & 41 & 0.35 & 19.19 & 26.20 & 0.34 & 0.04 & & & -- & -- & -- & -- & & -- & -- \\
\hline $42-44$ & 43 & 0.32 & 19.82 & 29.60 & 0.34 & 0.03 & & & 0.19 & 0.04 & $* *$ & -- & & 0.36 & 0.10 \\
\hline $44-46$ & 45 & 0.28 & 20.37 & 37.20 & 0.47 & 0.03 & & & -- & -- & -- & -- & & -- & -- \\
\hline $46-48$ & 47 & 0.19 & 20.76 & 43.60 & 0.50 & 0.03 & & & 0.35 & 0.10 & $* *$ & -- & & $\mathrm{Tr}$ & -- \\
\hline $48-50$ & 49 & 0.19 & 21.13 & 46.72 & 0.55 & 0.03 & & & -- & -- & -- & -- & & -- & -- \\
\hline $50-52$ & 51 & 0.24 & 21.62 & 47.20 & 0.46 & 0.04 & & & 0.33 & 0.09 & $* *$ & -- & & 0.32 & 0.19 \\
\hline Bottom & 53 & 0.14 & 21.89 & 35.99 & 1.49 & 0.02 & & & 0.45 & 0.09 & $* *$ & -- & & 0.84 & 0.24 \\
\hline
\end{tabular}

${ }^{1}$ Excess ${ }^{210} \mathrm{~Pb}$ Inventory $=9.0 \mathrm{dpm} / \mathrm{cm}^{2}$ (from top of core down to core interval $10-12 \mathrm{~cm}$ where excess ${ }^{210} \mathrm{~Pb}$ activity approaches a constant value) ${ }^{2}$ Inventory of ${ }^{17} \mathrm{Cs}=2.73 \mathrm{dpm} / \mathrm{cm}^{2}$. 
Table 9. Sample depths, loss-on-ignition, radionuclide activity, and inventory data, and ${ }^{210} \mathrm{~Pb}$ age calculations for core $99929-2$.

[Flux and inventory calculations completed by C.A. Rice; analytical data by M.E. Marot; --, not analyzed; **, not detected; Tr, Trace quantity, too low to quantify; dpm, disintegrations per minute]

\begin{tabular}{|c|c|c|c|c|c|c|c|c|c|c|c|c|c|c|c|c|}
\hline $\begin{array}{l}\text { Depth } \\
\text { (cm) }\end{array}$ & $\begin{array}{l}\text { Mean } \\
\text { depth } \\
(\mathrm{cm})\end{array}$ & $\begin{array}{l}\text { Dry bulk } \\
\text { density } \\
\left(\mathbf{g} / \mathrm{cm}^{3}\right)\end{array}$ & $\begin{array}{c}\text { Cumulative } \\
\text { sediment } \\
\text { weight } \\
\left(\mathbf{g} / \mathbf{c m}^{2}\right)\end{array}$ & $\begin{array}{l}\text { Loss-on- } \\
\text { ignition } \\
\text { (percent } \\
\text { dry wt.) }\end{array}$ & $\begin{array}{c}\text { Total }{ }^{210} \mathrm{~Pb} \\
\text { activity } \\
\text { (dpm/g) }\end{array}$ & $\begin{array}{c}\text { Total }{ }^{210} \mathrm{~Pb} \\
\text { activity } \\
\text { error } \\
( \pm \mathrm{dpm} / \mathrm{g})\end{array}$ & $\begin{array}{c}\text { Excess }{ }^{210} \mathrm{~Pb} \\
\text { activity } \\
\text { (dpm/g) }\end{array}$ & $\begin{array}{l}\text { Model } \\
\text { date }\end{array}$ & $\begin{array}{l}\text { Excess }{ }^{210} \mathrm{~Pb} \\
\text { inventory } \\
\left(\mathrm{dpm} / \mathrm{cm}^{2}\right)^{1}\end{array}$ & $\begin{array}{c}{ }^{226} \text { Ra activity } \\
\text { (dpm/g) }\end{array}$ & $\begin{array}{c}{ }^{226} \text { Ra activity } \\
\text { error } \\
( \pm \mathrm{dpm} / \mathrm{g})\end{array}$ & $\begin{array}{c}{ }^{137} \text { Cs activity } \\
\text { (dpm/g) }\end{array}$ & $\begin{array}{c}{ }^{137} \text { Cs activity } \\
\text { error } \\
( \pm \mathrm{dpm} / \mathrm{g})\end{array}$ & $\begin{array}{c}{ }^{137} \mathrm{Cs} \\
\text { inventory } \\
\left(\mathrm{dpm} / \mathrm{cm}^{2}\right)^{2}\end{array}$ & $\begin{array}{c}{ }^{234} \text { Th activity } \\
\text { (dpm/g) }\end{array}$ & $\begin{array}{c}{ }^{234} \text { Th activity } \\
\text { error } \\
( \pm \mathrm{dpm} / \mathrm{g})\end{array}$ \\
\hline $0-1$ & 0.5 & 0.076 & 0.076 & 32.95 & 31.47 & 0.58 & 29.41 & 1997 & 2.23 & -- & -- & -- & -- & -- & -- & -- \\
\hline $1-2$ & 1.5 & 0.161 & 0.237 & 29.79 & 27.80 & 0.46 & 25.74 & 1994 & 4.15 & -- & -- & -- & -- & -- & -- & -- \\
\hline $2-3$ & 2.5 & 0.218 & 0.455 & 26.60 & 24.20 & 0.34 & 22.14 & 1989 & 4.82 & 0.82 & 0.14 & 3.38 & 0.17 & 0.74 & 1.15 & 0.30 \\
\hline $3-4$ & 3.5 & 0.274 & 0.729 & 24.60 & 22.15 & 0.31 & 20.09 & 1984 & 5.50 & 0.95 & 0.15 & 3.62 & 0.17 & 0.99 & 1.30 & 0.33 \\
\hline $4-5$ & 4.5 & 0.264 & 0.992 & 23.60 & 18.81 & 0.28 & 16.75 & 1978 & 4.41 & 1.14 & 0.16 & 4.33 & 0.19 & 1.14 & 1.41 & 0.30 \\
\hline $5-6$ & 5.5 & 0.225 & 1.217 & 23.80 & 14.29 & 0.21 & 12.23 & 1973 & 2.75 & 1.13 & 0.16 & 4.75 & 0.20 & 1.07 & 1.59 & 0.37 \\
\hline 6-7 & 6.5 & 0.284 & 1.502 & 26.20 & 13.05 & 0.24 & 10.99 & 1967 & 3.13 & 1.28 & 0.16 & 5.96 & 0.22 & 1.70 & 1.76 & 0.38 \\
\hline $7-8$ & 7.5 & 0.241 & 1.743 & 30.00 & 13.85 & 0.30 & 11.79 & 1962 & 2.84 & 1.24 & 0.19 & 8.78 & 0.30 & 2.12 & 1.74 & 0.45 \\
\hline $8-9$ & 8.5 & 0.315 & 2.058 & 34.60 & 14.48 & 0.23 & 12.42 & 1956 & 3.91 & 1.39 & 0.18 & 8.10 & 0.27 & 2.55 & 1.89 & 0.36 \\
\hline $9-10$ & 9.5 & 0.240 & 2.298 & 35.00 & 13.67 & 0.23 & 11.61 & 1951 & 2.79 & 1.26 & 0.20 & 6.06 & 0.28 & 1.46 & 1.77 & 0.50 \\
\hline $10-12$ & 11.0 & 0.175 & 2.649 & 40.60 & 12.22 & 0.25 & 10.16 & 1943 & 3.56 & 1.23 & 0.13 & 2.21 & 0.13 & 0.77 & 1.81 & 0.31 \\
\hline $12-14$ & 13.0 & 0.283 & 3.216 & 29.20 & 6.11 & 0.11 & 4.05 & 1931 & 2.30 & 1.77 & 0.16 & 0.55 & 0.08 & 0.31 & 2.62 & 0.39 \\
\hline $14-16$ & 15.0 & 0.263 & 3.741 & 34.20 & 5.14 & 0.11 & 3.08 & 1920 & 1.62 & 1.47 & 0.12 & 0.19 & 0.05 & 0.10 & 2.09 & 0.27 \\
\hline $16-18$ & 17.0 & 0.295 & 4.330 & 37.60 & 3.88 & 0.08 & 1.82 & 1908 & 1.07 & 1.18 & 0.12 & $\mathrm{Tr}$ & -- & & 1.63 & 0.26 \\
\hline $18-20$ & 19.0 & 0.259 & 4.848 & 43.80 & 3.77 & 0.07 & 1.71 & 1897 & 0.89 & 0.95 & 0.10 & $* *$ & -- & & 1.08 & 0.21 \\
\hline $20-22$ & 21.0 & 0.277 & 5.403 & 24.40 & 2.06 & 0.04 & 0.00 & 1885 & 0.00 & -- & -- & -- & -- & & -- & -- \\
\hline $22-24$ & 23.0 & 0.323 & 6.049 & 37.00 & 3.28 & 0.12 & 1.22 & 1872 & 0.79 & 1.08 & 0.10 & $* *$ & -- & & 1.26 & 0.23 \\
\hline $24-26$ & 25.0 & 0.272 & 6.593 & 27.00 & 3.13 & 0.11 & 1.07 & 1860 & 0.58 & -- & -- & -- & -- & & -- & -- \\
\hline $26-28$ & 27.0 & 0.282 & 7.158 & 49.20 & 4.24 & 0.08 & 2.18 & 1848 & 1.23 & 0.69 & 0.07 & $* *$ & -- & & 1.15 & 0.16 \\
\hline $28-30$ & 29.0 & 0.223 & 7.603 & 40.32 & 4.50 & 0.09 & 2.44 & 1839 & 1.09 & -- & -- & -- & -- & & -- & -- \\
\hline $30-32$ & 31.0 & 0.322 & 8.247 & 37.60 & 3.71 & 0.07 & 1.65 & 1825 & 1.06 & 1.16 & 0.08 & $* *$ & -- & & 1.74 & 0.21 \\
\hline $32-34$ & 33.0 & 0.335 & 8.917 & 25.00 & 3.70 & 0.07 & 1.64 & 1811 & 1.10 & -- & -- & -- & -- & & -- & -- \\
\hline $34-36$ & 35.0 & 0.369 & 9.655 & 25.60 & 4.13 & 0.09 & 2.07 & 1796 & 1.53 & 1.18 & 0.10 & $* *$ & -- & & 1.67 & 0.26 \\
\hline $36-38$ & 37.0 & 0.331 & 10.318 & 36.80 & 4.41 & 0.13 & 2.35 & 1782 & 1.56 & -- & -- & -- & -- & & -- & -- \\
\hline $38-40$ & 39.0 & 0.152 & 10.622 & 70.60 & 14.87 & 0.26 & 12.81 & 1775 & 3.90 & 0.57 & 0.12 & $* *$ & -- & & 1.09 & 0.30 \\
\hline $40-42$ & 41.0 & 0.153 & 10.928 & 76.80 & 18.17 & 0.36 & 16.11 & 1769 & 4.92 & -- & -- & -- & -- & & -- & -- \\
\hline $42-44$ & 43.0 & 0.196 & 11.320 & 63.80 & 9.93 & 0.21 & 7.87 & 1761 & 3.09 & 0.42 & 0.07 & $* *$ & -- & & 0.83 & 0.17 \\
\hline Bottom & 44.5 & 0.112 & 11.543 & 53.80 & 12.22 & 0.24 & 10.16 & 1756 & 2.27 & 0.76 & 0.12 & 1.19 & 0.09 & & 1.16 & 0.26 \\
\hline
\end{tabular}

${ }^{210} \mathrm{~Pb}$ Excess Inventory $=48.58 \mathrm{dpm} / \mathrm{cm}^{2}$ (from top of core down to core interval $26-28 \mathrm{~cm}$, that is 150 years)

${ }^{2}$ Inventory of ${ }^{137} \mathrm{Cs}=12.95 \mathrm{dpm} / \mathrm{cm}^{2}$. 
Table 10. Sample depths, loss-on-ignition, radionuclide activity, and inventory data, and ${ }^{210} \mathrm{~Pb}$ age calculations for core $99929-3$.

[Flux and inventory calculations completed by C.A. Rice; analytical data by M.E. Marot; -- not analyzed; **, not detected; Tr, trace quantity, too low to quantify; dpm, disintegrations per minute]

\begin{tabular}{|c|c|c|c|c|c|c|c|c|c|c|c|c|c|c|c|c|}
\hline $\begin{array}{l}\text { Depth } \\
\text { (cm) }\end{array}$ & $\begin{array}{l}\text { Mean } \\
\text { depth } \\
\text { (cm) }\end{array}$ & $\begin{array}{l}\text { Dry bulk } \\
\text { density } \\
\left(\mathrm{g} / \mathrm{cm}^{3}\right)\end{array}$ & $\begin{array}{c}\text { Cumulative } \\
\text { sediment } \\
\text { weight } \\
\left(\mathbf{g} / \mathbf{c m}^{2}\right) \\
\end{array}$ & $\begin{array}{c}\text { Loss-on- } \\
\text { ignition } \\
\text { (percent } \\
\text { dry wt.) }\end{array}$ & $\begin{array}{c}\text { Total }{ }^{20} \mathbf{P b} \\
\text { activity } \\
\text { (dpm/g) }\end{array}$ & $\begin{array}{c}\text { Total }{ }^{210} \mathrm{~Pb} \\
\text { activity } \\
\text { error } \\
( \pm \mathrm{dpm} / \mathrm{g})\end{array}$ & $\begin{array}{c}\text { Excesss } 210 \mathrm{~Pb} \\
\text { activity } \\
(\mathrm{dpm} / \mathrm{g})^{1,2}\end{array}$ & $\begin{array}{l}\text { Model } \\
\text { date }\end{array}$ & $\begin{array}{l}\text { Excesss }{ }^{210} \mathrm{~Pb} \\
\text { inventory } \\
\left(\mathrm{dpm} / \mathrm{cm}^{2}\right)^{3}\end{array}$ & $\begin{array}{l}{ }^{226} \text { Ra activity } \\
\text { (dpm/g) }\end{array}$ & $\begin{array}{c}{ }^{226} \text { Ra activity } \\
\text { error } \\
( \pm \mathrm{dpm} / \mathrm{g})\end{array}$ & $\begin{array}{c}{ }^{137} \mathrm{Cs} \text { activity } \\
(\mathrm{dpm} / \mathrm{g})\end{array}$ & $\begin{array}{c}{ }^{137} \text { Cs activity } \\
\text { error } \\
( \pm \mathrm{dpm} / \mathrm{g})\end{array}$ & $\begin{array}{c}{ }^{137} \mathrm{Cs} \text { inventory } \\
\left(\mathrm{dpm} / \mathrm{cm}^{2}\right)^{4}\end{array}$ & $\begin{array}{l}{ }^{234} \mathrm{Th} \text { activity } \\
\text { (dpm/g) }\end{array}$ & $\begin{array}{c}{ }^{234} \text { Th activity } \\
\text { error } \\
( \pm \mathrm{dpm} / \mathrm{g})\end{array}$ \\
\hline $\begin{array}{ll}0-1 \\
\end{array}$ & 0.5 & 0.058 & 0.058 & 18.40 & 13.47 & 0.20 & 11.55 & 1997 & 0.669 & 1.92 & 0.17 & 3.87 & 0.16 & 0.22 & $\mathrm{Tr}$ & -- \\
\hline $1-2$ & 1.5 & 0.231 & 0.289 & 15.80 & 12.28 & 0.22 & 10.87 & 1990 & 2.511 & 1.41 & 0.21 & 2.96 & 0.19 & 0.68 & 1.15 & 0.35 \\
\hline $2-3$ & 2.5 & 0.319 & 0.608 & 13.20 & 10.43 & 0.28 & 8.39 & 1981 & 2.672 & 2.05 & 0.22 & 5.86 & 0.25 & 1.87 & 1.35 & 0.37 \\
\hline $3-4$ & 3.5 & 0.403 & 1.010 & 12.20 & 8.46 & 0.14 & 5.88 & 1968 & 2.368 & 2.58 & 0.18 & 6.52 & 0.22 & 2.63 & 1.30 & 0.35 \\
\hline $4-5$ & 4.5 & 0.555 & 1.565 & 8.60 & 6.03 & 0.09 & 3.63 & 1952 & 2.014 & 2.40 & 0.16 & 3.55 & 0.12 & 1.97 & 1.77 & 0.24 \\
\hline $5-6$ & 5.5 & 0.453 & 2.017 & 12.20 & 3.95 & 0.07 & 1.71 & 1938 & 0.775 & 2.23 & 0.18 & 1.50 & 0.10 & 0.68 & 1.11 & 0.26 \\
\hline 6-7 & 6.5 & 0.286 & 2.303 & 15.20 & 3.33 & 0.07 & 0.60 & 1929 & 0.171 & 2.73 & 0.31 & 1.09 & 0.18 & 0.31 & 1.08 & 0.48 \\
\hline $7-8$ & 7.5 & 0.260 & 2.563 & 15.60 & 2.60 & 0.06 & 0.04 & 1921 & 0.009 & 2.56 & 0.23 & 0.52 & 0.11 & 0.14 & 1.02 & 0.39 \\
\hline $8-9$ & 8.5 & 0.261 & 2.824 & 13.80 & 2.19 & 0.05 & -0.21 & 1913 & 0.000 & 2.40 & 0.28 & $\mathrm{Tr}$ & -- & & $\operatorname{Tr}$ & -- \\
\hline $9-10$ & 9.5 & 0.328 & 3.153 & 12.40 & 2.36 & 0.05 & 0.20 & 1903 & 0.000 & 2.16 & 0.20 & $* *$ & -- & & $\mathrm{Tr}$ & -- \\
\hline $10-12$ & 11.0 & 0.328 & 3.808 & 13.00 & 2.13 & 0.05 & 0.85 & 1884 & 0.558 & 1.27 & 0.09 & 0.06 & 0.03 & & 0.32 & 0.11 \\
\hline $12-14$ & 13.0 & 0.282 & 4.373 & 12.80 & 1.19 & 0.04 & 0.65 & 1866 & 0.366 & 0.54 & 0.07 & $* *$ & -- & & 0.45 & 0.12 \\
\hline $14-16$ & 15.0 & 0.271 & 4.915 & 14.60 & 1.06 & 0.03 & 0.54 & 1850 & 0.290 & 0.53 & 0.08 & $* *$ & -- & & 0.59 & 0.17 \\
\hline $16-18$ & 17.0 & 0.238 & 5.392 & 11.75 & 0.92 & 0.02 & 0.38 & 1836 & 0.182 & 0.54 & 0.09 & $* *$ & -- & & 0.66 & 0.18 \\
\hline $18-20$ & 19.0 & 0.253 & 5.897 & 11.25 & 0.82 & 0.02 & 0.36 & 1820 & 0.184 & 0.46 & 0.08 & $* *$ & -- & & 0.49 & 0.15 \\
\hline $20-22$ & 21.0 & 0.332 & 6.562 & 29.00 & 0.75 & 0.02 & 0.15 & 1800 & 0.097 & -- & -- & -- & -- & & -- & -- \\
\hline $22-24$ & 23.0 & 0.186 & 6.935 & 43.20 & 1.61 & 0.03 & 0.87 & 1789 & 0.325 & 0.74 & 0.10 & $* *$ & -- & & 1.34 & 0.21 \\
\hline $24-26$ & 25.0 & 0.170 & 7.274 & 43.80 & 1.40 & 0.03 & 0.73 & 1779 & 0.248 & -- & -- & -- & -- & & -- & -- \\
\hline $26-28$ & 27.0 & 0.194 & 7.661 & 59.60 & 1.76 & 0.04 & 1.17 & 1767 & 0.451 & 0.59 & 0.08 & $* *$ & -- & & 0.99 & 0.17 \\
\hline $28-30$ & 29.0 & 0.156 & 7.974 & 61.60 & 1.80 & 0.05 & 1.17 & 1757 & 0.366 & -- & -- & -- & -- & & -- & -- \\
\hline $30-32$ & 31.0 & 0.175 & 8.324 & 58.60 & 1.92 & 0.05 & 1.26 & 1747 & 0.443 & 0.66 & 0.09 & ** & -- & & 1.09 & 0.22 \\
\hline $32-34$ & 33.0 & 0.181 & 8.686 & 49.60 & 1.60 & 0.04 & 0.96 & 1736 & 0.348 & -- & -- & -- & -- & & -- & -- \\
\hline $34-36$ & 35.0 & 0.283 & 9.251 & 49.40 & 1.76 & 0.09 & 1.13 & 1719 & 0.641 & 0.62 & 0.07 & $* *$ & -- & & 0.88 & 0.16 \\
\hline $36-38$ & 37.0 & 0.354 & 9.958 & 24.60 & 0.90 & 0.02 & 0.35 & 1697 & 0.250 & -- & -- & -- & -- & & -- & -- \\
\hline $38-40$ & 39.0 & 0.170 & 10.297 & 61.40 & 1.67 & 0.04 & 1.20 & 1687 & 0.406 & 0.47 & 0.07 & ** & -- & & 1.17 & 0.16 \\
\hline $40-42$ & 41.0 & 0.162 & 10.622 & 67.50 & 1.70 & 0.04 & 1.27 & 1677 & 0.412 & -- & -- & -- & -- & & -- & -- \\
\hline $42-44$ & 43.0 & 0.153 & 10.928 & 65.25 & 1.73 & 0.04 & 1.34 & 1668 & 0.409 & 0.39 & 0.12 & $* *$ & -- & & 0.41 & 0.24 \\
\hline $44-46$ & 45.0 & 0.247 & 11.422 & 64.40 & 1.89 & 0.05 & 1.44 & 1653 & 0.711 & -- & -- & -- & -- & & -- & -- \\
\hline $46-48$ & 47.0 & 0.129 & 11.679 & 62.40 & 1.50 & 0.04 & 0.98 & 1645 & 0.252 & 0.52 & 0.11 & ** & -- & & 0.77 & 0.25 \\
\hline $48-50$ & 49.0 & 0.198 & 12.076 & 60.60 & 1.19 & 0.03 & 0.69 & 1633 & 0.272 & -- & -- & -- & -- & & -- & -- \\
\hline $50-52$ & 51.0 & 0.198 & 12.472 & 64.20 & 1.50 & 0.04 & 1.01 & 1621 & 0.399 & 0.49 & 0.07 & $* *$ & -- & & 0.81 & 0.15 \\
\hline $52-54$ & 53.0 & 0.189 & 12.850 & 60.80 & 1.22 & 0.03 & 0.65 & 1610 & 0.246 & -- & -- & -- & -- & & -- & -- \\
\hline $54-56$ & 55.0 & 0.196 & 13.241 & 65.00 & 1.67 & 0.03 & 1.03 & 1598 & 0.404 & 0.64 & 0.07 & ** & -- & & 0.81 & 0.16 \\
\hline $56-58$ & 57.0 & 0.165 & 13.572 & 65.20 & 1.56 & 0.04 & 0.96 & 1588 & 0.317 & -- & -- & -- & -- & & -- & -- \\
\hline $58-60$ & 59.0 & 0.189 & 13.950 & 60.80 & 1.53 & 0.04 & 0.93 & 1576 & 0.352 & 0.60 & 0.09 & $* *$ & -- & & 0.92 & 0.21 \\
\hline $60-62$ & 61.0 & 0.202 & 14.355 & 56.00 & 2.34 & 0.06 & 1.742 & 1564 & 0.705 & & -- & -- & -- & & -- & -- \\
\hline Bottom & 63.5 & 0.275 & 14.905 & -- & -- & -- & -- & -- & & -- & -- & $* *$ & -- & & 1.49 & 0.22 \\
\hline
\end{tabular}




\section{${ }^{210} \mathrm{~Pb}$ Radiometric Calculations}

The chemical systematics affecting the accumulation of ${ }^{210} \mathrm{~Pb}$ in the Animas River watershed require that the radionuclides be in equilibrium with ambient ${ }^{226} \mathrm{Ra}$, and assume that there is a constant flux of excess ${ }^{210} \mathrm{~Pb}, \mathrm{f}\left(\mathrm{dpm} / \mathrm{cm}^{2} / \mathrm{yr}\right)$, and mass of sediment, $\mathrm{r}\left(\mathrm{g} / \mathrm{cm}^{2} / \mathrm{yr}\right)$. Figures 4-6, which are loglinear plots of total ${ }^{210} \mathrm{~Pb}$ versus cumulative sediment weight, indicate these assumptions are reasonable considering the type of environment. Perturbations from straight-line decay of ${ }^{210} \mathrm{~Pb}$ are primarily from variations in organic content in the cores, which trap the radionuclides more effectively. Because of the influence of ground water in lower portions of some of the cores and the variable and sometimes high organic content of the cores, the excess ${ }^{210} \mathrm{~Pb}$, which is the difference between total ${ }^{210} \mathrm{~Pb}$ and ${ }^{226} \mathrm{Ra}$, was calculated using constant values of ${ }^{226} \mathrm{Ra}$ in two of the cores. In the other core, the difference between total ${ }^{210} \mathrm{~Pb}$ and the measured ${ }^{226} \mathrm{Ra}$ was used.

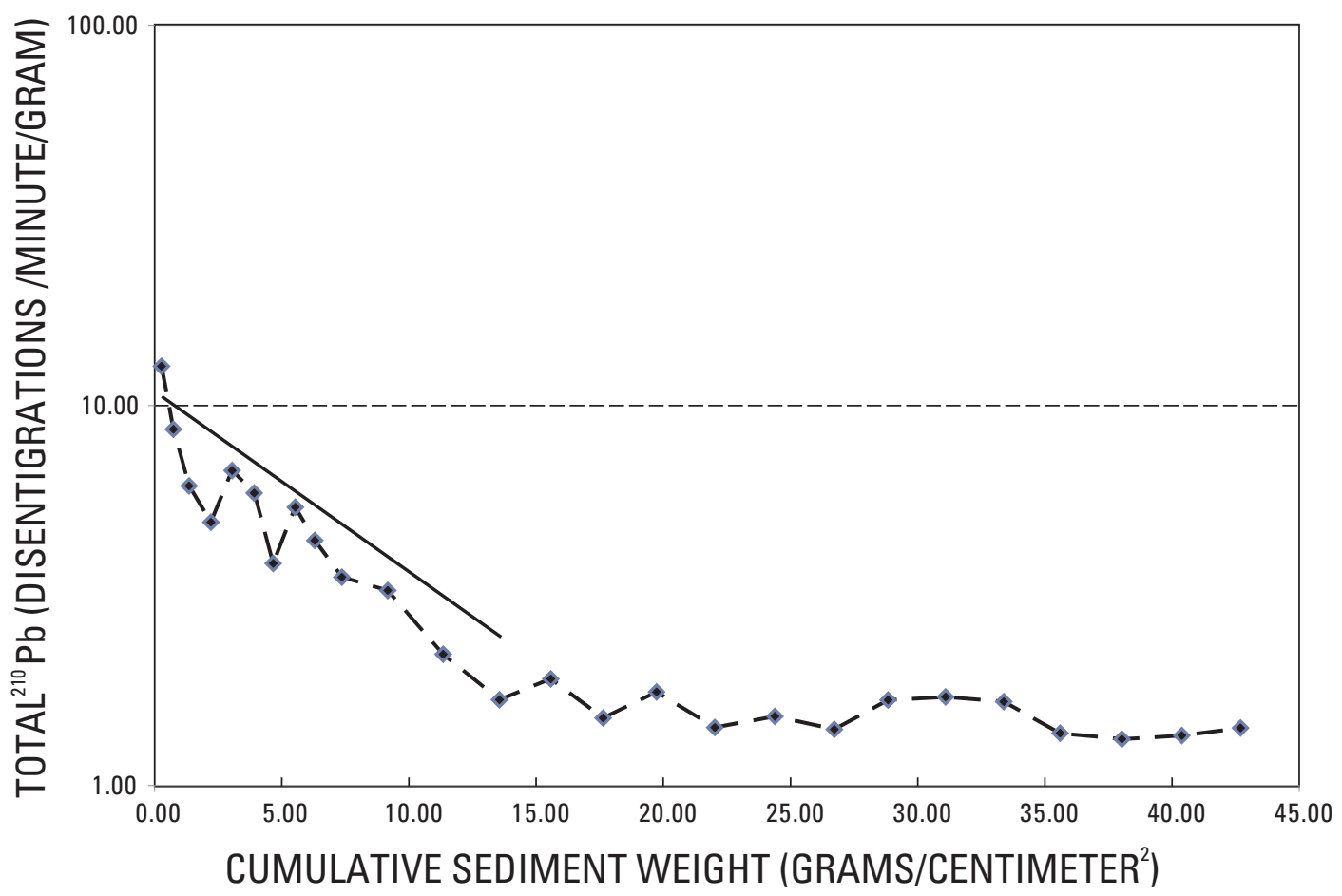

Figure 4. Log-linear plot of total ${ }^{210} \mathrm{~Pb}$ activity versus the cumulative sediment weight for core $99928-1$ (table 7). Straight line shows exponential decay of ${ }^{210} \mathrm{~Pb}$ assuming constant rates of supply of ${ }^{210} \mathrm{~Pb}$ and mass. 


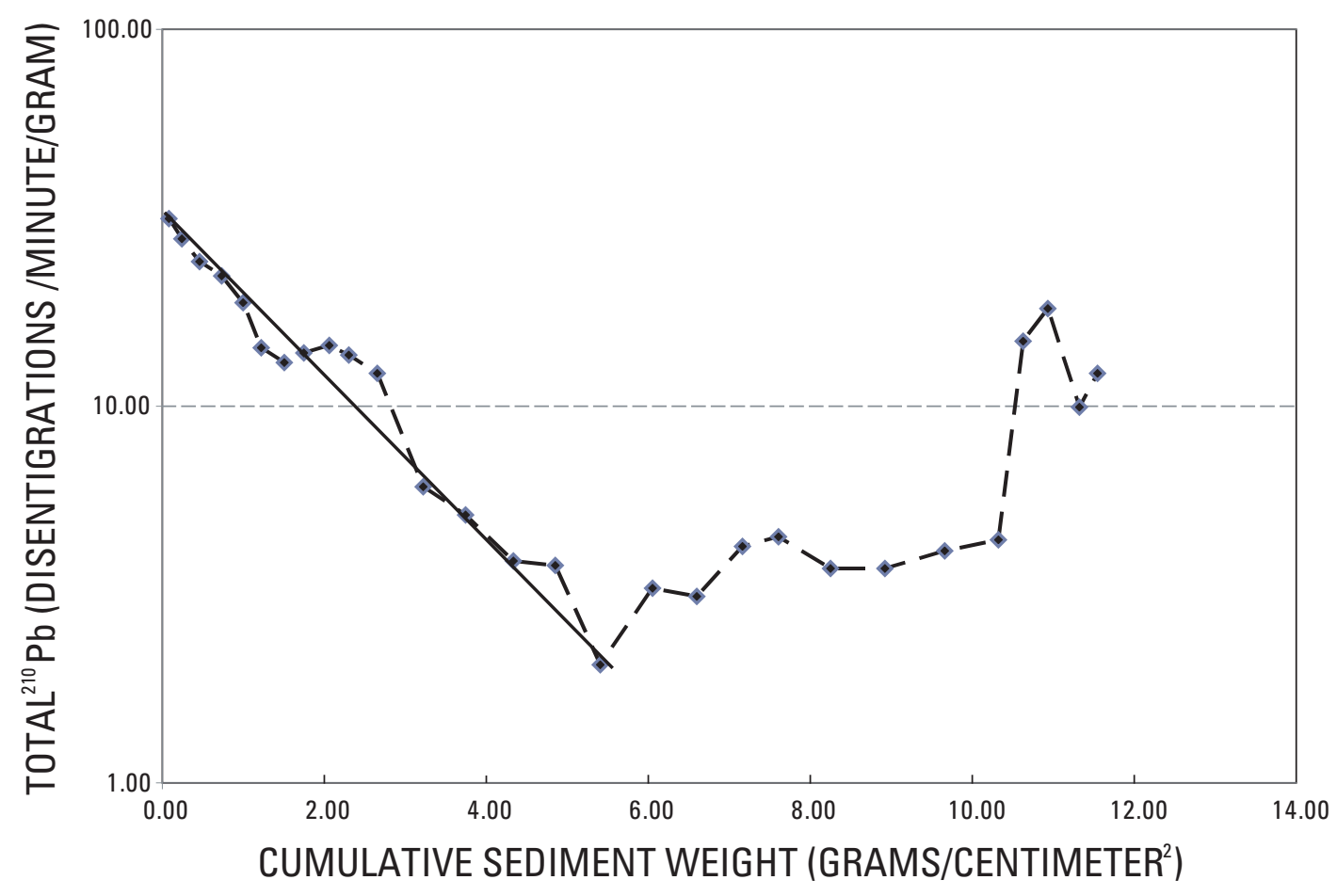

Figure 5. Log-linear plot of total ${ }^{210} \mathrm{~Pb}$ activity versus the cumulative sediment weight for core 99929-2 (table 9). Straight line shows exponential decay of ${ }^{210} \mathrm{~Pb}$ assuming constant rates of supply of ${ }^{210} \mathrm{~Pb}$ and mass.

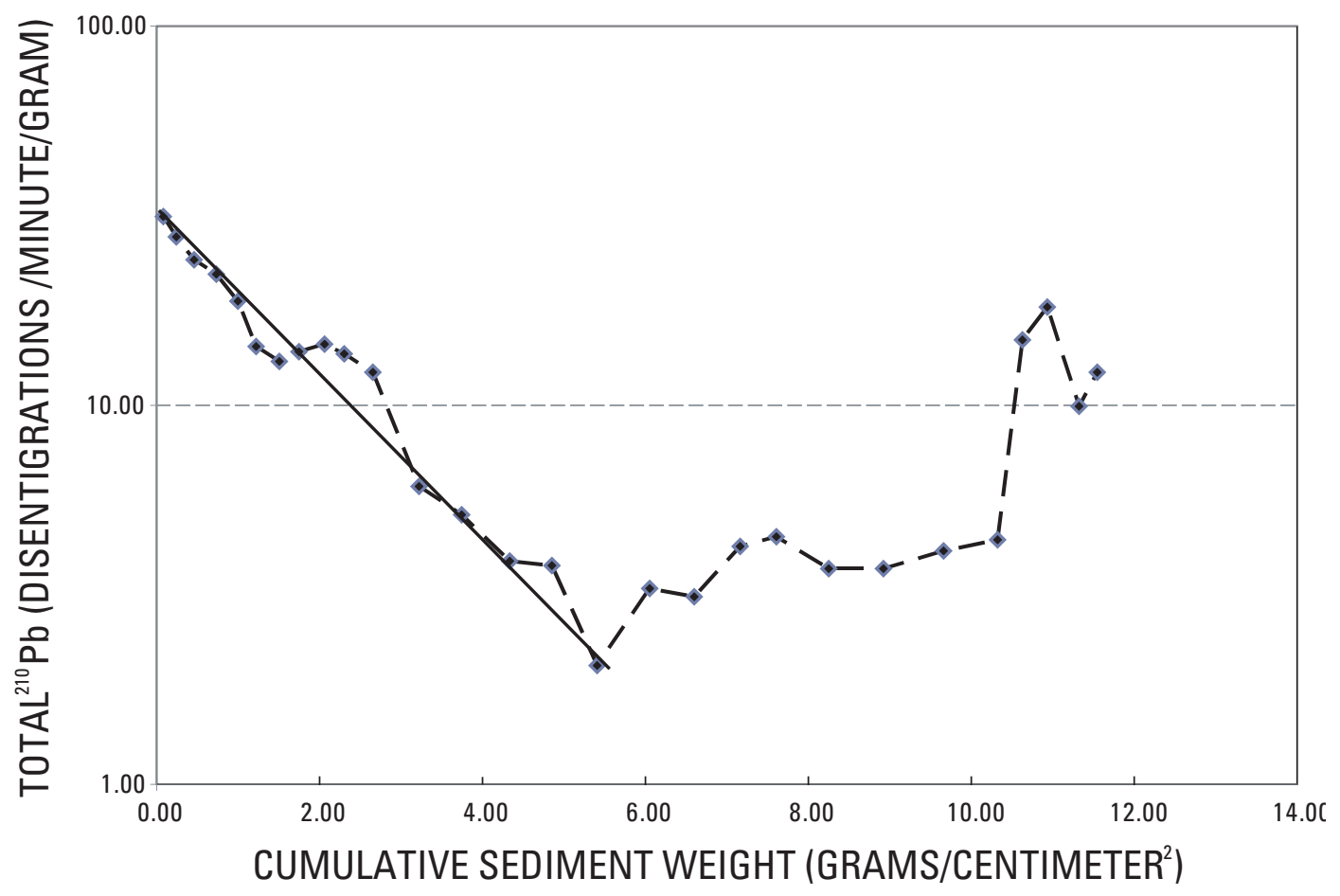

Figure 6. Log-linear plot of total ${ }^{210} \mathrm{~Pb}$ activity versus the cumulative sediment weight for core 99929-3 (table 10). Straight line shows exponential decay of ${ }^{210} \mathrm{~Pb}$ assuming constant rates of supply of ${ }^{210} \mathrm{~Pb}$ and mass. 
The excess activity $\left(\mathrm{A}_{\mathrm{ex}}\right)$ of ${ }^{210} \mathrm{~Pb}$ is the result of the exponential decay of the excess ${ }^{210} \mathrm{~Pb}$ at time zero, $\mathrm{A}_{\mathrm{o}}: \mathrm{A}_{\mathrm{ex}}=\mathrm{A}_{\mathrm{o}} \mathrm{e}^{-\lambda t}$, where $\lambda$ is the radioactive decay constant $\left(\lambda=0.03114 \mathrm{yr}^{-1}\right)$, $A_{o}=f / r$, and $t=g / r$, where $g\left(g / \mathrm{cm}^{2}\right)$ is the cumulative weight of sediment at a given depth. Ages of sediment can be calculated from $\mathrm{g} / \mathrm{r}$. By using the cumulative sediment weight rather than core depth, effects of compaction are taken into account. Statistical counting errors given in tables 7-10 are the major source of error. For this study, samples were counted for a period of time to provide less than 5 percent counting error, thus the calculated ages have a \pm 5 percent error. This translates to \pm 2.5 years at 50 years B.P.

Individual cores were analyzed by calculating a best-fit curve to the excess ${ }^{210} \mathrm{~Pb}$ data and the cumulative sediment weight using an unweighted Marquardt-Levenberg parameter optimization method (Press and others, 1989). A distinct advantage of this procedure over other methods used to reduce ${ }^{210} \mathrm{~Pb}$ data is that all data points are used in the sediment-accumulation-rate calculations, including negative values (which are the result of statistical counting errors in the deeper parts of the sediment column where there is a small signal-to-noise ratio). This method has the further advantage of eliminating the mathematical consequence that produces "old age errors" and a null ${ }^{210} \mathrm{~Pb}$ disequilibria that is inherent in other best-fit interpolation routines. Null ${ }^{210} \mathrm{~Pb}$ disequilibria mathematically yield an infinitely old age, a geologic impossibility. Analytical results and an alternative age model for core 99929-2 are presented in table 9.

The ${ }^{210} \mathrm{~Pb}$ age model for each core was compared with the ${ }^{137} \mathrm{Cs}$ activity peak, which was assumed to be $1963 \pm 2$ years. The calculated ${ }^{210} \mathrm{~Pb}$ ages agree well with the age calculated from the ${ }^{137} \mathrm{Cs}$ activity peak.

\section{Geochemical Influence of Periodic Flooding of the Oxbow Lakes, Durango Area}

Water level records for the lower Animas River reach indicate that the oxbow lakes in the Durango, Colo., area are occasionally flooded in the spring whenever the flow exceeded 8,000 cubic feet per second (cfs). Flood stages are tabulated in table 11 from stream flow data obtained largely from the stream gage at the 32nd Street Bridge located immediately downstream from the site (fig. 2). Observations of the water level during the May 2005 flood showed that a flow of 8,000 cfs resulted in bank-full flow at the site (fig. 2). On the basis of these flow records, flood deposition of sediment from overbank sheet flow in these oxbow lakes should not have occurred since 1949 , and had probably only occurred 8 to 10 times between 1897 and 1949 (table 11). The largest flood events were the 500-year flood in 1911 and two large floods in 1927. Because the floodplain area in which the oxbow lakes reside is flat and marshy, detritus from local erosion deposited in the oxbow lakes would be minimal. Overbank flow sampled during the May 2005 flood shows that the river had a very small suspended-sediment load by the time it had reached 8,000 cfs. Thus, the inorganic sediment deposited within the oxbow lake is dominated by wind-blown detritus derived largely from sediment on the exposed riverbed at low flow. 
Table 11. Discharge records from NWIS gage 09361500, 32nd St. Bridge, Durango, Colo.

\begin{tabular}{ccc}
\hline $\begin{array}{c}\text { Flood } \\
\text { year }\end{array}$ & $\begin{array}{c}\text { Dates discharge } \\
\mathbf{>}, \mathbf{0 0 0} \text { cfs } \\
\text { (no records from this gage } \\
\text { during period-in italics) }\end{array}$ & $\begin{array}{c}\text { Peak discharge } \\
\text { ft/sec } \\
\text { (estimated peak }\end{array}$ \\
\hline 1909 & Sept. 24 & 10,000 \\
1911 & Oct. 5-11 & 25,000 \\
1914 & June 2 & 8,330 \\
1917 & June 14-24 & 8,460 \\
1920 & May 21-June 2 & 9,260 \\
1921 & June 10-16 & 9,300 \\
1922 & June 10 & 7,000 \\
1927 & June 29 20,000 \\
1927 & Sept. 10-13 & 14,000 \\
1941 & May 13-16 & 9,500 \\
1948 & May 20 & 7,500 \\
1949 & June 18-21 & 10,700 \\
1952 & June 8-11 & 7,550 \\
1957 & June 5,6 & 8,090 \\
1970 & Sept. 6 & 7,400 \\
1973 & June 11, 12 & 7,110 \\
1979 & May 28-30 & 7,480 \\
1979 & June 7 & 7,260 \\
1980 & June 10-12 & 7,550 \\
1984 & May 24, 25 & 7,070 \\
1985 & June 9 & 7,250 \\
2005 & May 22-25 & 8,070 \\
\hline & &
\end{tabular}




\section{References Cited}

Briggs, P.H., 2002, The determination of forty elements in geological and botanical samples by inductively coupled plasma-atomic emission spectrometry, chap. G of Taggart, J.E., ed., Analytical methods for chemical analysis of geologic and other materials: U.S. Geological Survey Open-File Report 02-223, 18 p.

Buxton, H.T., Nimick, D.A., von Guerard, Paul, Church, S.E., Frazier, Ann, Gray, J.R., Lipin, B.R., Marsh, S.P., Woodward, Daniel, Kimball, Briant, Finger, Susan, Ischinger, Lee, Fordham, J.C., Power, M.S., Bunck, Christine, and Jones, J.W., 1997, A science-based, watershed strategy to support effective remediation of abandoned mine lands: Fourth Annual International Conference on Acid Rock Drainage Proceedings, v. IV, Vancouver, B.C., Canada, May 31-June 5, 1997, p. 1,869-1,880.

Church, S.E., 1981, Multielement analysis of fifty-four geochemical reference samples using inductively coupled plasma-atomic emission spectrometry: Geostandards Newsletter, v. 5, p. 133-160.

Church, S.E., Mosier, E.L., and Motooka, J.M., 1987, Mineralogical basis for the interpretation of multielement (ICP-AES), oxalic acid, and aqua-regia partial digestions of stream sediments for reconnaissance exploration geochemistry: Journal of Geochemical Exploration, v. 29, p. 207-233.

Church, S.E., von Guerard, Paul, and Finger, S.E., 2007, eds., Integrated investigations of environmental effects of historical mining in the Animas River watershed, San Juan County, Colorado: U.S. Geological Survey Professional Paper 1651, 1,096 p.

Crock, J.G., Lichte, F.E., and Briggs, P.H., 1983, Determination of elements in National Bureau of Standards geologic reference materials SRM 278 obsidian and SRM 688 basalt by inductively coupled plasma-atomic emission spectroscopy: Geostandards Newsletter, v. 7, p. 335-340.

Cutshall, N.H., Larsen, I.L., and Olsen, C.R., 1983, Direct analysis of ${ }^{210} \mathrm{~Pb}$ in sediment samples: Self-adsorption corrections: Nuclear Instrumental Methods Physics Research, v. 26, p. 309-312.

Ferderer, D.A., 1996, National overview of abandoned mine land sites utilizing the minerals availability system (MAS) and geographic information systems (GIS) technology: U.S. Geological Survey Open-File Report 96-549, 42 p.

Fey, D.L., Unruh, D.M., and Church, S.E., 1999, Chemical data and lead isotopic compositions in stream-sediment samples from the Boulder River watershed, Jefferson County, Montana: U.S. Geological Survey Open-File Report 99-575, 147 p. [http://pubs.usgs.gov/of/1999/ofr-99-0575/].
Fey, D.L., Church, S.E., and Unruh, D.M., 2000, Geochemical and lead isotopic data from sediment cores, fluvial tailings, iron bogs, and pre-mining terrace deposits, Animas River watershed, Colorado, 1995-1999: U.S. Geological Survey Open-File Report 2000-0465, 13 p. [http://pubs.usgs.gov/ of/2000/ofr-00-0465/].

Flynn, W.W., 1968, The determination of polonium-210 in environmental materials: Analytica Chimica Acta, v. 43, p. 121-131.

Jones, W.R., 2007, History of mining and milling practices and production in San Juan County, Colorado, 1871-1991, in Church, S.E., von Guerard, Paul, and Finger, S.E., eds., Integrated investigations of environmental effects of historical mining in the Animas River watershed, San Juan County, Colorado: U.S. Geological Survey Professional Paper 1651, p. 39-86.

National Institute of Standards and Technology (NIST), 1993a, Certificate of analysis standard reference material 2704, Buffalo River sediment: National Institute of Standards and Technology.

National Institute of Standards and Technology (NIST), 1993b, Certificate of analysis standard reference material 2709, San Joaquin soil: National Institute of Standards and Technology.

National Institute of Standards and Technology (NIST), 1993c, Certificate of analysis standard reference material 2710, contaminated Montana soil: National Institute of Standards and Technology.

National Institute of Standards and Technology (NIST), 1993d, Certificate of analysis standard reference material 2711, Montana soil: National Institute of Standards and Technology.

Press, W.H., Flannery, B.P., Teukolsky, S.A., and Vetterling, V.T., 1989, Numerical recipes: The art of scientific computing: New York, Cambridge University Press, 234 p.

Robbins, J.A., Holmes, C.W., Halley, R.B., Bothner, M.A., Shinn, E.A., Graney, J., Keeler, G., TenBrink, M., Orlandini, K.A., and Rudnick, D.E., 2000, Time-averaged flux of lead and fallout radionuclides to sediment in Florida Bay: Journal of Geophysical Research, v. 105, p. 28,805-28,821.

Runnells, D.D., Shephard, T.A., and Angino, E.E., 1992, Metals in water-Determining natural background concentrations of dissolved components in water at mining, milling, and smelting sites: Mining Engineering, v. 50, p. 65-71.

Taggart, J.E., Jr., Lindsay, J.R., Scott, B.A., Vivit, D.V., Bartel, A.J., and Stewart, K.C., 1987, Analysis of geologic materials by wavelength-dispersive X-ray fluorescence spectrometry, in Baedecker, P.A., ed., Methods for geochemical analysis: U.S. Geological Survey Bulletin 1770, p. E1-E19. 
USDA Forest Service and Bureau of Land Management, 2007, Abandoned mine lands: A decade of progress reclaiming hardrock mines: Forest Service Publication No. FS-891 and BLM Publication No. BLM-WO-GI-07-013-3720, 33 p. [http://www.fs.fed.us/geology/aml-index.htm and http:// www.blm.gov/aml].

U.S. Geological Survey, 1898, Topographic map of the Durango quadrangle: U.S. Geological Survey, scale: 1:250,000.
U.S. Geological Survey, 1997, A plan for the USGS abandoned mine lands initiative, 1997-2001: U.S. Geological Survey web page [http://amli.usgs.gov/amli/5year/, accessed February 15, 2007].

Wilson, S.A., Briggs, P.H., Mee, J.S., and Siems, D.F., 1994, Determination of thirty-two major and trace elements in three NIST soil SRMs using ICP-AES and WDXRF: Geostandards Newsletter, v. 18, p. 58-89.

Publishing support provided by:

Denver Publishing Service Center

For more information concerning this publication, contact:

Team Chief Scientist, USGS Central Mineral Resources

Box 25046, Mail Stop 973

Denver, CO 80225

(303)236-1562

Or visit the Central Mineral Resources Team Web site at:

http://minerals.cr.usgs.gov/ 
